\title{
An evolutionary discretized Lambert approach for optimal long-range rendezvous considering impulse limit
}

\author{
Abolfazl Shirazi ${ }^{\mathrm{a}, \mathrm{b}, *}$, Josu Ceberio $^{\mathrm{b}}$, Jose A. Lozano ${ }^{\mathrm{a}, \mathrm{b}}$ \\ ${ }^{a}$ Basque Center for Applied Mathematics BCAM, Bilbao, 48009, Spain \\ ${ }^{b}$ University of the Basque Country UPV/EHU, Donostia, 20018, Spain
}

\begin{abstract}
In this paper, an approach is presented for finding the optimal long-range space rendezvous in terms of fuel and time, considering limited impulse. In this approach, the Lambert problem is expanded towards a discretized multi-impulse transfer. Taking advantage of an analytical form of multi-impulse transfer, a feasible solution that satisfies the impulse limit is calculated. Next, the obtained feasible solution is utilized as a seed for generating individuals for a hybrid selfadaptive evolutionary algorithm to minimize the total time, without violating the impulse limit while keeping the overall fuel mass the same as or less than the one associated with the analytical solution. The algorithm eliminates similar individuals and regenerates them based on a combination of Gaussian and uniform distribution of solutions from the fuel-optimal region during the optimization process. Other enhancements are also applied to the algorithm to make it auto-tuned and robust to the initial and final orbits as well as the impulse limit. Several types of the proposed algorithm are tested considering varieties of rendezvous missions. Results reveal that the approach can successfully reduce the overall transfer time in the multi-impulse transfers while minimizing the fuel mass without violating the impulse limit. Furthermore, the proposed algorithm has superior performance over standard evolutionary algorithms in terms of convergence and optimality.
\end{abstract}

\footnotetext{
${ }^{*}$ Corresponding author

Email addresses: ashirazi@bcamath.org (Abolfazl Shirazi), josu.ceberio@ehu.eus (Josu Ceberio), jlozano@bcamath.org (Jose A. Lozano)
}

Preprint submitted to Aerospace Science and Technology

DOI: $10.1016 /$ j.ast.2019.105400 
Keywords: Discretized Lambert problem, Long-range rendezvous,

Self-adaptive algorithm, Impulse limit, Evolutionary approach, Optimal transfer

\section{Introduction}

Rendezvous orbital dynamics is a key operational element for complicated space missions, which has a research history of several decades, and many novel research ideas and results on this topic are still appearing in different missions such as asteroids explorations [1], Mars missions [2] and Earth orbit transfers [3]. The general long-range rendezvous problems are usually solved based on the Lambert method which has been one of the most extensively studied methods for decades and still is a subject of interest in many researches $[4,5]$. In a long-range rendezvous, the spacecraft is expected to have an orbital maneuver where, in the general case, all of the orbital elements involved suffer changes. This type of noncoplanar orbit transfer problem is the early phase of the overall space rendezvous [6]. In this type of mission, a two-impulse transfer obtained by the Lambert method that starts on the initial orbit and ends on the final orbit within a specific time can be the fuel-optimal transfer. However, for some specific cases, such as bi-elliptic Hohmann transfers, it is analytically possible to have the same or less fuel consumption than a two-impulse transfer for specific missions [7]. In either cases, no observation is applied to the magnitude of impulses, and thus, the solutions might not be feasible in scenarios where impulse limits are considered. In fact, the problem becomes more challenging when propulsion systems with low impulses are used in such non-coplanar transfers. When the goal is to find the optimal transfer in terms of fuel and time complying a given impulse limit, finding the global optimal solution becomes challenging. One effective option to deal with this difficulty is to use meta-heuristics and evolutionary algorithms $[8,9]$. As a result, a more thorough investigation is needed to find the best solution using these numerical methods and techniques [10].

In recent years, several attempts have been made to deal with impulsive 
transfers in various space missions and conditions with different techniques $[11,12]$. Such conditions or constraints can be the actuation uncertainties [13], collision avoidance constraint $[14,15]$ or other criteria. According to the literature, this multi-objective problem generally includes two types of costs and two types of constraints. The costs are fuel and time while the initial and final orbits along with allowable impulse limit are considered as constraints. Besides the impulse magnitude constraint, impulse direction is also sometimes considered as another kind of constraint in the literature. It can be the tangential impulse [16], along-track impulse [17] or continuous-thrust transfer with specific control magnitude and direction constraints [18]. Regarding the initial and final condition, the problems can be categorized as point to point, point to orbit and orbit to orbit maneuvers. A lot of research has been dedicated to such problems. However, an efficient approach in facing the problem is that which can be used regardless of the problem type. In [19], the problem of multi-impulse transfer is tackled by an analytical solution based on polynomials. However, the transfer time is not considered, and the approach is tested on special cases. Coplanar two-impulse rendezvous is studied in [20] and [21]. The research in [22] focused on a homotopic targeting technique for space rendezvous. Although it adequately considered the presence of orbital perturbations, the impulse limit is not taken into account. More research can also be found in the literature in which either the time, the impulse limit or other criteria have been taken into consideration besides the fuel consumption [23, 24]. Impulsive rendezvous between non-coplanar orbits considering fuel, time and impulse limits makes the problem multi-objective and challenging to solve. To that end, a metaheuristic algorithm combined with an analytical solution of the multi-impulse transfer has been considered. The developed approach minimizes both the fuel and time, keeping the minimum necessary number of impulses without violating the impulse limit in a long-range rendezvous mission. Particularly, the proposed strategy in the current context is a direct approach based on the discretized Lambert problem and a novel hybrid self-adaptive evolutionary algorithm. In this approach, as the first step, the problem is solved, disregarding the impulse 
limit and transfer time and a solution that minimizes the total fuel consumption is achieved. This solution can be obtained either analytically or by means of an NLP or an evolutionary algorithm. Having this solution, the Lambert problem associated with the obtained transfer trajectory is extended to multiimpulse transfers in which the overall transfer is divided into a specific number of stages. Then, an analytical scheme is proposed based on dividing the velocity increments into the necessary number of small impulses at the intersections of space orbits corresponding to each stage. This solution results in a specific sequence of impulses within the proposed approach, which is feasible in terms of fuel consumption. Considering this solution as a seed solution for generating individuals with high quality, a robust self-adaptive evolutionary algorithm is proposed. The algorithm benefits from several enhancements over the standard evolutionary techniques by hybridizing an NLP solver with a modified Particle Swarm Optimization. The algorithm is constructed as a self-adaptive technique since its parameters are auto tuned according to the orbital parameters of the initial and final orbits as well as the specified impulse limit for the space rendezvous mission. Combined with the proposed approach, the algorithm is tested on a wide set of long-range space rendezvous missions with various impulse limits. Results indicate that the algorithm is capable of decreasing the overall transfer time while it satisfies the impulse limit and holds the optimal fuel consumption.

The rest of the paper is organized as follows. Section 2 introduces the discretized Lambert problem for multi-impulse orbit transfers. The simple feasible solution, obtained by an analytical approach is proposed in Section 3 based on dividing the velocity increments within the intersections of space orbits. In Section 4, the robust self-adaptive evolutionary algorithm, along with the improvements in its structure, are discussed. Section 5 provides the simulation results obtained by utilizing the proposed approach in several long-range space rendezvous missions. Finally, the conclusions are provided in Section 6 . 


\section{Discretized Lambert Problem}

\subsection{The Approach}

Consider a general long-range rendezvous with initial and final orbital elements as $p_{0}=\left[a_{0}, e_{0}, i_{0}, \Omega_{0}, \omega_{0}, \nu_{0}\right]$ and $p_{f}=\left[a_{f}, e_{f}, i_{f}, \Omega_{f}, \omega_{f}, \nu_{f}\right]$. Since two orbits do not have any intersections and no orbital elements are the same between the two orbits, a two-impulse transfer trajectory that minimizes the total fuel consumption exists. The transfer trajectory starts from the initial true anomaly of $\theta_{i}$ of the initial orbit and ends in the final true anomaly of $\theta_{f}$ of the final orbit. These two anomalies correspond with two radius vectors $\vec{r}_{i}$ and $\vec{r}_{f}$. Besides these two anomalies, a third parameter fulfills a complete Lambert problem regarding any desired approach. The third parameter can be semimajor axis [25] (Lagrange transfer-time equation), eccentricity [26] (Avanzini's approach), a universal variable [27] (Izzo's approach), flight-path angle [28] or any other parameter that constructs a unique Lambert problem. Considering the Lagrange transfer-time equation, the unknown set of variables can be established as $x=\left[\begin{array}{lll}\theta_{i} & \theta_{f} & t\end{array}\right]$ when only the orbit transfer is desired. If the actual rendezvous is also desired besides the orbit change, the unknown variables will be $x=\left[\begin{array}{ll}\theta_{i} & t\end{array}\right]$ with respect to the relative phase difference of chaser and target. In either case, the problem can be turned into a blackbox optimization problem with total velocity increment $\Delta v$ as the output, which is the summation of two impulses at intersections $\Delta v=\Delta v_{1}+\Delta v_{2}$.

By means of an effective nonlinear programming (NLP) method or an evolutionary algorithm (EA), the best solution with minimum fuel could be approximated. However, no penalty is considered for the excessive magnitude of $\Delta v_{i}$ when low-impulse propulsion systems are utilized. In order to find the solution with minimum fuel and transfer time in the multi-impulse maneuver with respect to a given impulse limit, a new approach is proposed as shown in Fig. 1.

In this approach, the original Lambert problem is divided into $N$ number of stages. Each stage represents a unique Lambert problem with unknown 


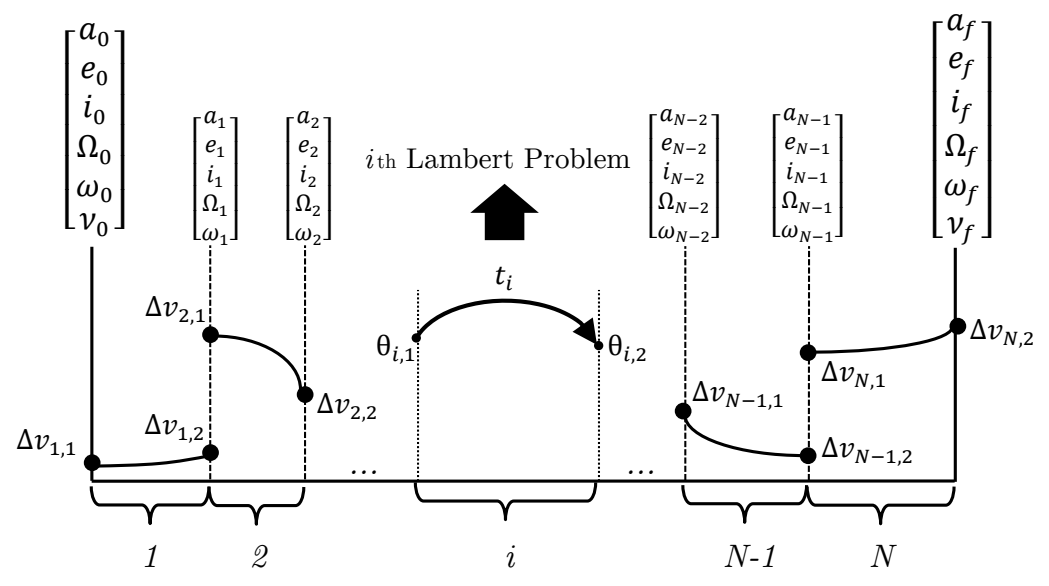

Figure 1: Scheme of discretized Lambert problem.

Lambert problem variables. Having $N$ number of stages will generate $N-1$ intermediate orbits, represented by $a_{i}, e_{i}, i_{i}, \Omega_{i}$ and $\omega_{i}(i=1$ to $N-1)$, along with $N$ jumps. Every jump is denoted by initial and final anomalies as $\theta_{i, 1}$ and $\theta_{i, 2}$ corresponding to the initial and final state vectors and the transfer time $t_{i}$ in each stage. By considering the orbital elements of stages and the Lambert problem variables as the inputs, a complete multi-impulse Lambert problem with $2 N$ impulses $\left(\Delta v_{i, 1}\right.$ and $\left.\Delta v_{i, 2}\right)$ will be fulfilled. Therefore, the decision variables, denoted by the vector $x$, will be formed as:

$$
x=x\left(a_{i}, e_{i}, i_{i}, \Omega_{i}, \omega_{i}, \theta_{i, j}, t_{i, j}\right)
$$

According to this approach, a total of $2 N-1$ sets of orbital elements will be known when a solution is achieved. These sets contain $N-1$ intermediate orbits, which are the inputs of the problem, and $N$ jumps, which are trajectories representing the solution from each stage that represents a minor Lambert problem. The $3 N$ number of variables are associated with $N$ Lambert problems, and $5(N-1)$ variables are associated with the shape and orientation of the $N-1$ intermediate orbits. Therefore, a total of $8 N-5$ decision variables are associated with the optimization of multi-impulse transfer in this approach 
for $N$ stages. Recalling that each stage has two impulses, the total number of variables for $n$ number of impulses $(N=2 n)$ will be $4 n-5$.

The initial and terminal conditions can be easily handled for various types of long-range rendezvous missions in this approach. This can be done by adjusting the initial true anomaly of the first stage $\left(\theta_{1,1}\right)$ and the final true anomaly of the last stage $\left(\theta_{N, 2}\right)$. As for orbit to orbit rendezvous, the initial true anomalies of two spacecraft $\nu_{0}$ and $\nu_{f}$ are unknown and therefore $\theta_{1,1}$ and $\theta_{N, 2}$ are free. In this case, the problem will be tackled according to the described optimization variables. For orbit to point rendezvous, when it is necessary for the two spacecraft to be in the same true anomaly in the final orbit, the parameter $\nu_{f}$ is known, which is the initial position of the target spacecraft in the final orbit at epoch. In this case, $\theta_{N, 2}$ will be fixed and its value can be calculated based on the total coast times and the initial true anomaly of the target spacecraft $\left(\theta_{N, 2}=\theta_{N, 2}\left(\nu_{f}, t_{i}, \bar{t}_{i}\right)\right)$. Similarly, if it is required that the first impulse of the chaser occurs at a specific true anomaly in the initial orbit ( $\nu_{0}$ is known), the variable $\theta_{1,1}$ will be fixed. To sum up, depending on what sort of long-range rendezvous is the subject of the problem, the two optimization variables $\left(\theta_{1,1}\right.$ and $\left.\theta_{N, 2}\right)$ can be either fixed or free.

This approach has some advantages over the traditional methods. First, as with most of the multi-impulse approaches based on the Lambert problem [29], the total number of inputs is lower in comparison to the traditional approach in which the direction, magnitude and time of impulses are considered as the decision variables [30] (total of $4 n$ decision variables). As an example, in the traditional approach for a two-impulse transfer, the time of acting for each impulse along with the impulse vector, including the magnitude and two angles representing the direction of the impulse in three dimensions are considered as the decision variables. However, in the current approach, only three variables are required including the initial true anomaly, the final true anomaly and the transfer time. Although the number of variables is lower, the approach comes with the burden of tackling the Lambert problem in achieving the solution, which requires iterations. This is due to the fact that, by defining multiple minor 
Lambert problems, the majority of the characteristics of the transfer trajectories wil be revealed. In the other words, the shape of the transfer trajectories are taken into account instead of the impulse directions in the Cartesian coordinate system. Regarding this fact, this approach can be referred to as an impulsive shape-based approach.

The next advantage is handling the terminal conditions. The initial and final condition for point to point, point to orbit and orbit to orbit cases can be easily handled in the current approach by setting the Lambert problem variables as free or fixed in the first and last stages. However, satisfying the terminal condition in the traditional approach is an issue which usually needs to be considered as an additional term in the objective function. Besides, since the shape of stages is defined via the actual orbital elements with physical meanings, the method benefits from rapid convergence as the orbital elements have known boundaries in real applications.

\subsection{The Objectives}

As the boundary conditions are already satisfied by the proposed approach, three types of objectives are defined for the problem including fuel, time and impulse violation. Regarding the proposed approach, the overall fuel consumption in terms of $\Delta v$ in every stage is denoted by $J_{f}$ and is defined as:

$$
J_{f}=\sum_{i=1}^{N}\left(\Delta v_{i, 1}+\Delta v_{i, 2}\right)
$$

The total transfer time is the summation of all coasting times between the impulses. Regarding the proposed approach, two types of coasting times exist, denoting by $t_{i}$ and $\bar{t}_{i}$. The first type $\left(t_{i}\right)$ is the time associated with each minor Lambert problem in every stage between $\Delta v_{i, 1}$ and $\Delta v_{i, 2}$. The latter $\left(\bar{t}_{i}\right)$ is the time between the impulses $\Delta v_{i, 2}$ and $\Delta v_{i+1,1}$, in which the spacecraft travels between two sequential Lambert problem. Therefore, the time objective, represented by $J_{t}$, is defined as: 


$$
J_{t}=\sum_{i=1}^{N} t_{i}+\sum_{i=1}^{N-1} \bar{t}_{i}
$$

The impulse violation regarding a given impulse limit needs to be calculated for each $\Delta v$ in every stage. As a result, the penalty denoted by the $j$ th $\Delta v$ in $i$ th stage is calculated as

$$
J_{i, j}=\frac{1+\operatorname{sgn}\left(\Delta v_{i, j}-\eta\right)}{2}\left(\Delta v_{i, j}-\eta\right)
$$

where $\eta$ is the given allowable impulse during the orbit transfer. In this equation, the typical sign function is used to extract the appropriate penalty for each impulse. According to this equation, if the impulse is less than the predefined limit $\left(\Delta v_{i, j}<\eta\right)$, the penalty associated with that impulse becomes zero $\left(J_{i, j}=\right.$ $0)$. On the other hand, if the impulse exceeds the predefined limit $\left(\Delta v_{i, j}>\right.$ $\eta$ ), the associated penalty will be equal to the amount of exceeded impulse magnitude $\left(J_{i, j}=\Delta v_{i, j}-\eta\right)$.

Consequently, the overall magnitude of the penalty function due to the impulse violations in all stages is calculated as:

$$
J_{v}=\sum_{i=1}^{N}\left(J_{i, 1}+J_{i, 2}\right)
$$

Having the cost functions, the overall objective function can be written via scalarizing the three objectives as:

$$
J=J_{f}+\zeta J_{v}+\xi J_{t}
$$

where $\zeta$ and $\xi$ are scalarization coefficients for impulse violation and transfer time respectively. In the literature, the impact of the choice of the underlying scalarizing coefficients is still far from being well understood in space orbit design and optimization problems. Due to this matter, it is very important and crucial to choose these parameters according to the type of the space transfer. To demonstrate the effect of these weighting coefficients, one example of feasible 
and non-feasible solutions within the solution domain of a sample four-impulse rendezvous with one intermediate orbit is represented in Fig. 2 and Fig. 3. In this case, an orbit to orbit transfer with the initial and final orbits as $p_{0}=$ $[10000,0.1,30,40,55]$ and $p_{f}=[16000,0.4,25,50,30]$ is considered with impulse limit of $\eta=0.5 \mathrm{~km} / \mathrm{s}$.

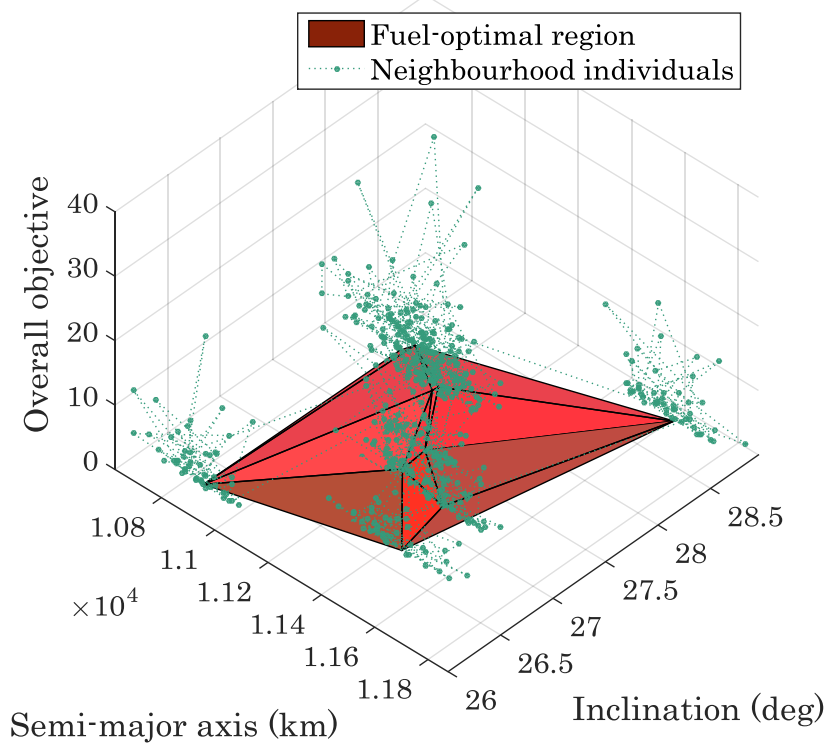

Figure 2: Solution domain of $J(\xi=0)$ in a four-impulse rendezvous $(\Delta v=1.4777 \mathrm{~km} / \mathrm{s})$.

In Fig. 2 the solutions are plotted as a function of semi-major axis and inclination of the intermediate orbit. In this figure, the surface associated with a set of good solutions (obtained by means of the approach in Sec. II and IV) is plotted, which includes the solutions with minimum fuel in this case. In addition, neighboring solutions of the surface are plotted as green points. Note that the time objective function has been disregarded $(\xi=0)$. The points correspond to the solutions with larger objective values either due to impulse violation or fuel consumption. Extracting the points near the minimum-fuel region with respect to a selective threshold and recalculating $J$ and $J_{t}$ for $\xi \neq 0$ are shown in Fig. 3. In this figure, the objective representing the total transfer times $\left(J_{t}\right)$ for fuel-optimal region versus the overall cost is depicted. 


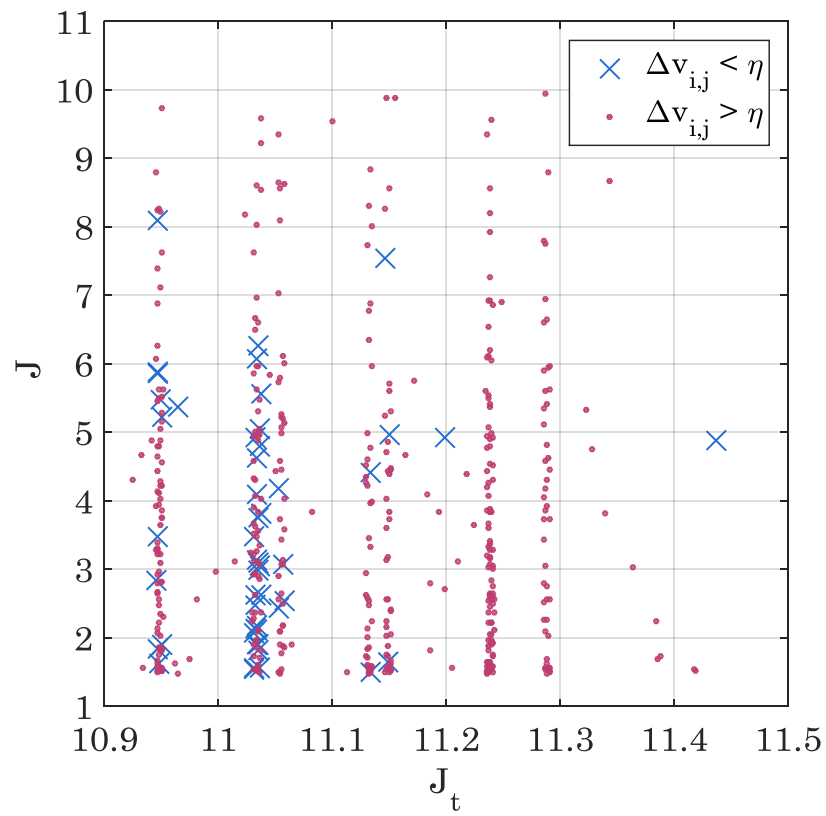

Figure 3: Time-optimal solutions in the neighborhood of fuel-optimal region.

In this figure, the solutions for acceptable transfers satisfying the impulse limit are plotted along with non-feasible solutions in this matter. Local optima regions can be obviously distinguished in Fig. 3 showing that the consideration of $\zeta$ significantly affects the desired region and reaching the global optimal solution is challenging in this type of problem. It is worth noting that the best values of $\zeta$ are different from case to case. The major challenge is that the tuned value of this parameter along with $\xi$ for one space rendezvous does not necessarily enhance the search process in another rendezvous problem. Therefore, these parameters have to be tuned automatically according to the initial and final orbits in each rendezvous mission. Such self-adaptive concepts makes the approach robust to every unique Lambert problem with a given impulse limit.

\section{Simple Feasible Solution}

Regarding the proposed approach, the problem can be solved via an EA. However, two issues will arise. First, the solution domain of the optimization 
problem becomes too large when a high number of impulses are desired. The reason is that by considering very low-impulse transfers, the number of stages is increased which consequently increases the number of optimization variables. Following this, the landscape of the problem becomes chaotic, which makes the convergence process slow and the quality of the final solution can drop dramatically. The second issue is that the minimum number of impulses required for the entire transfer is unknown.

In order to deal with these issues, individuals near the solution which minimized the fuel consumption can be used to improve the quality of the initial populations of the EA and also to calculate the necessary number of impulses regarding the given impulse limit. Seeding the EA based on a feasible solution derived from the fuel-optimal region will effectively improve the convergence and the optimality of the algorithm. This is due to the fact that the newly generated populations have small values for one or two objectives while satisfying the impulse limit, forcing the algorithm to minimize the time near the fuel-optimal region. The seeding technique can be used either within the initial population at the beginning of the optimization, or during the optimization process when the diversity of the population is less than a predefined threshold, or both. To implement this concept, the problem is solved for $N_{0}$ number of stages without considering time $(\xi=0)$ and impulse violation $(\zeta=0)$ initially. This solution can be derived from either an existing analytical approach or a numerical solution. Having the solution for $N=N_{0}$, the $\Delta v_{i, j}$ at the intersection of transfer orbit with $N_{i}$ th and $N_{i+1}$ th intermediate orbits can be divided into a necessary number of minor impulses. A schematic view of this concept is depicted in Fig. 4.

Following this process, the impulse at each intersection is divided into minor ones keeping the impulse direction fixed. The required number of divisions can be calculated based on the given impulse limit $\eta$ as:

$$
\varphi_{i, j}=\left\lceil\frac{\Delta v_{i, j}}{\eta}\right\rceil
$$




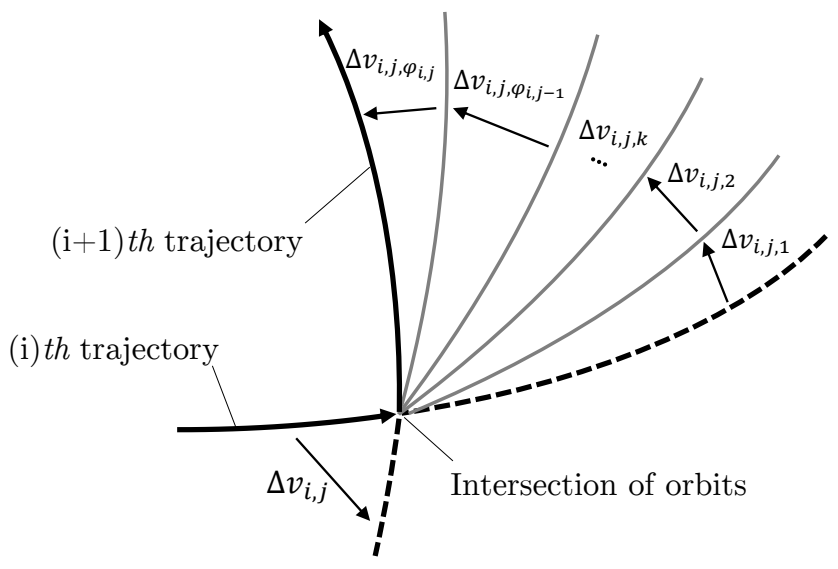

Figure 4: Impulse division at intersection of orbits.

where $\varphi_{i, j}$ is the minimum number of required impulses. Accordingly, the actual impulse division at intersections for $1<k<\varphi_{i, j}$ can be represented by:

$$
\sum_{k=1}^{\varphi_{i, j}} \Delta v_{i, j, k}=\Delta v_{i, j}
$$

where $\Delta v_{i, j, k}$ is the $k$ th minor impulse in the $j$ th intersection of orbits in the $i$ th stage. The current division keeps the impulse direction fixed as $\Delta \vec{v}_{i, i, k} /\left|\Delta \vec{v}_{i, i, k}\right|=$ $\Delta \vec{v}_{i, j} /\left|\Delta \vec{v}_{i, j}\right|$ and it satisfies $\Delta v_{i, j, k}=\Delta v_{i, j} / \varphi_{i, j}$ if the desired impulses are equally divided. Calculating the orbital elements based on the newly obtained velocity vectors and rearranging them back to the discretized Lambert problem in the previous section will end in the solutions satisfying the given impulse limit. The solution obtained here will be used as a candidate solution to be utilized by the developed EA in the following section. Considering the fact that the EA starts with a random distribution of individuals within the known boundary limits, to minimize the overall transfer time, one can take advantage of univariate Gaussian distribution and sample individuals near the fuel-optimal region for seeding the algorithm instead of uniform random distribution. 


\section{Hybrid Self-Adaptive Evolutionary Algorithm}

The algorithm for solving the multi-impulse discrete Lambert approach is described in this section. Particularly, a hybrid self-adaptive algorithm that combines an enhanced evolutionary algorithm with the feasible solution is presented. During the development of the EA, the features of the space rendezvous mission are utilized to make the approach robust to any changes in the space mission. In this section, three components are presented briefly, describing the main enhancements that have been taken into account. The aim of this section is to present a layout of the connection between the algorithm parameters and the elements of space rendezvous, which are utilized to make the algorithm self-adaptive. Details regarding each modification are omitted and the reader is urged to refer to the references provided.

\subsection{Hybridization of Algorithms}

According to the presented approach, the search space of the discretized Lambert problem is a continuous domain, possibly with a variable number of local optima depending on the type of mission. Since the goal is to reach the global optimal solution considering three objectives in general, there is a high possibility for the solution to get trapped in the local optima regions. In order to take advantage of the swarm intelligence and the shape of the landscape of the problem and also compensate the weaknesses of stochastic and gradientbased methods, hybridization of methods from two different types of algorithms has been taken into account. In recent years, such hybrid evolutionary algorithms have been well developed in different spacecraft trajectory design and optimization problems [31].

The core of the optimization algorithm in this approach is based on an Improved Particle Swarm Optimization (IPSO), hybridized with an NLP solver. IPSO performs searching via a swarm of particles that updates from iteration to iteration considering some enhancements. To seek the optimal solution, each

particle moves in the direction to its previously best (pbest) position and the 
global best (gbest) position in the swarm [16]. One has

$$
\begin{aligned}
\operatorname{pbest}(i, j) & =\underset{k=1, \ldots, j}{\arg \min }\left[J\left(x_{i}(k)\right)\right], \quad i \in\left\{1,2, \ldots, N_{p}\right\} \\
\operatorname{gbest}(j) & =\underset{\substack{i=1, \ldots, N_{p} \\
k=1, \ldots, j}}{\arg \min }\left[J\left(x_{i}(k)\right)\right]
\end{aligned}
$$

where $i$ here denotes the particle index, $N_{p}$ the total number of particles, and $j$ the current iteration number. The velocity $\hat{v}$ and position $\hat{p}$ of particles are updated by the following equations:

$$
\begin{aligned}
& \hat{v}_{i}(j+1)=w_{i}(j) v_{i}(j)+c_{1} \delta_{1}\left(\text { pbest }(i, j)-x_{i}(j)\right)+c_{2} \delta_{2}\left(\text { gbest }(j)-x_{i}(j)\right) \\
& x_{i}(j+1)=x_{i}(j)+\hat{v}_{i}(j+1)
\end{aligned}
$$

where $\hat{v}$ denotes the velocity, $w_{i}(j)$ is the inertia weight used to balance the global exploration and local exploitation, $\delta_{1}$ and $\delta_{2}$ are uniformly distributed random numbers within range $[0,1]$, and $c_{1}$ and $c_{2}$ are personal and global learning coefficients.

The inertia weight $w_{i}(j)$ is to bring about a balance between the exploration and exploitation characteristics of the process. A large inertia weight facilitates a global search while a small inertia weight facilitates a local search. By changing the inertia weight dynamically, the search capability is dynamically adjusted. In this algorithm, $w_{i}(j)$ is defined as [32].

$$
w_{i}(j+1)= \begin{cases}\min \left(1, w_{i}(j)+\left(1-w_{0}\right) \times\left(\exp \left(\frac{(x i(j+1)-p b e s t(i, j))^{2}}{-2 \sigma^{2}}\right)+\rho\right)\right) & \text { if } \delta_{i}(j)>0 \text { and } \delta_{i}(j+1)>0 \\ \max \left(0.1, w_{i}(j)-w_{0} \times\left(1-\exp \left(\frac{(x i(j+1)-p b e s t(i, j))^{2}}{-2 \sigma^{2}}\right)-\rho\right)\right) & \text { else if } \delta_{i}(j)<0 \text { and } \delta_{i}(j+1)<0 \\ w_{i}(j) & \text { otherwise }\end{cases}
$$

where $w_{0}$ is the initial inertia weight which is considered equal for all particles in all dimensions, in the $(j+1)$ th iteration. The Gaussian kernel width $(\sigma)$ is adjusted in a way that covers the maximum movement of the particles. $\rho$ is a small positive number used to ensure a proper increase or decrease of the inertia 
weight. $\delta_{i}$ is the feedback parameter defined as:

$$
\delta_{i}(j+1)=\left\{\begin{aligned}
1 & \text { if } J\left(x_{i}(j)\right)<\operatorname{pbest}(i, j-1) \\
-1 & \text { else }
\end{aligned}\right.
$$

According to this equation, the last two steps during the course of the run are analyzed to be sure of making decisions about the value of the inertia weight. When a particle succeeds in some sequential steps, it will have more tendency to memorize its direction. Probably because it will have more success in this direction. When a particle does not succeed in some sequential steps, it has less tendency to memorize its previous direction. Probably because it will have no more success in this direction.

In every generation within the process of optimization, the position of the particles $x_{i}(j)$ is improved with an NLP, leading to fast convergence. LBFGS [33], an approximation to BFGS, which requires a lot less memory is used as an efficient NLP for this matter. The position of the $i$ th particle is improved in the $j$ th iteration as:

$$
x_{i}(j+1)=x_{i}(j)-\alpha_{j} H_{j} \nabla J\left(x_{i}(j)\right)
$$

where $\alpha_{j}$ is the step length and $H_{j}$ is updated at every iteration by means of the formula

$$
H_{j+1}=V_{j}^{T} H_{j} V_{j}+\rho_{j} s_{j} s_{j}^{T}
$$

where

$$
\begin{aligned}
\rho_{j} & =\frac{1}{y_{j}^{T} s_{j}} \\
V_{j} & =I-\rho_{j} y_{j} s_{j}^{T}
\end{aligned}
$$


and

$$
\begin{aligned}
& s_{j}=x_{i}(j+1)-x_{i}(j) \\
& y_{j}=\nabla f_{j+1}-\nabla f_{j}
\end{aligned}
$$

Since the inverse Hessian approximation $H_{j}$ will generally be dense, the cost of storing and manipulating it is prohibitive when the number of variables is large due to high number of impulses in the rendezvous mission. To circumvent this problem, a modified version of $H_{j}$ is utilized implicitly, by storing a certain number $(\lambda)$ of the vector pairs $\left(s_{j}, y_{j}\right)$ used in the formulas Eq. 16 to Eq. 20. The product $H_{j} \nabla J\left(x_{i}(j)\right)$ can be obtained by performing a sequence of inner products and vector summations involving $\nabla J\left(x_{i}(j)\right)$ and the pairs $\left(s_{j}, y_{j}\right)$. After the new iterate is computed, the oldest vector pair in the set of pairs $\left(s_{j}, y_{j}\right)$ is replaced by the new pair obtained from the current step in Eq. 19 and Eq. 20. In this way, the set of vector pairs includes curvature information from the most recent iterations. Practical experience has shown that modest values of $\lambda$ often produce satisfactory results. The algorithm also benefits from other modifications as well, such as mirror effect and velocity clamping. The reader may refer to $[34,35,36]$ for the details.

\subsection{Generating Near-optimal Transfers}

The proposed analytical seeding is utilized for generating the populations for the developed EA. In order not to lose the diversity of the populations, uniform random distribution of individuals is also utilized alongside the Gaussian random distribution to reach the optimal transfer. The new population $x_{n}$ is then generated as a vector of mixed individuals. Assuming the generation of $n$ individuals $\left(x_{n}\right)$, the algorithm generates $\epsilon \times n$ individuals based on the uniform distribution $\left(x_{u}\right)$ and produces $(1-\epsilon) \times n$ individuals based on Gaussian distribution $\left(x_{g}\right)$ near the region in the search space which has the same minimized-fuel as in the feasible solution as: 


$$
x_{g} \sim \mathcal{N}\left(x^{*}, \sigma^{2}\left(p_{0}, p_{f}, N\right)\right)
$$

where $x^{*}$ is the feasible solution with the minimized fuel the same as the solution with $N_{0}$ stages, which satisfies the impulse limit and $\sigma^{2}$ is the selective variances. One example of such a distribution is illustrated in Fig. 5.

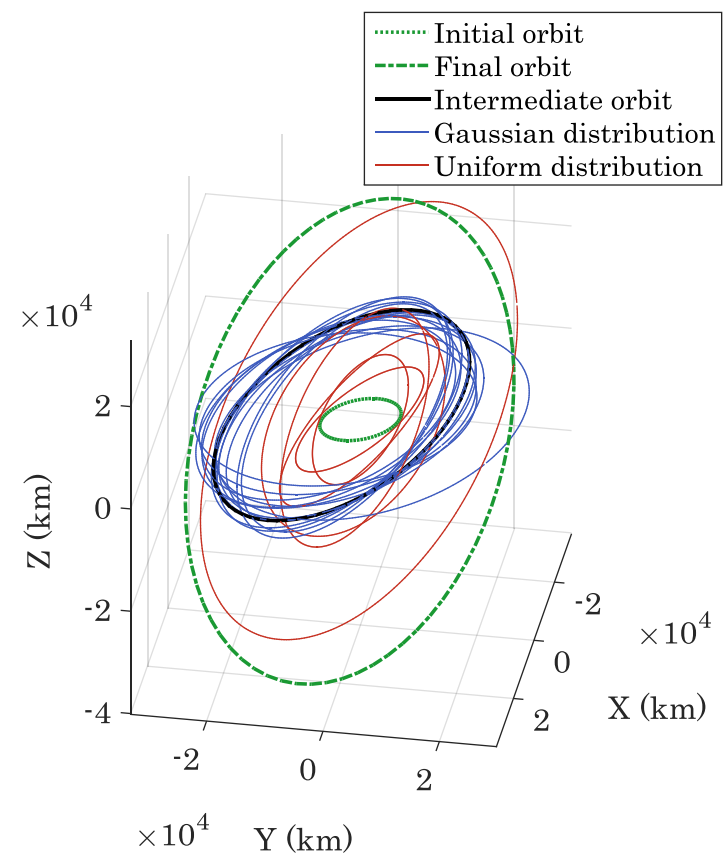

Figure 5: Distribution orbits in four-impulse rendezvous $(\epsilon=0.4)$.

Fig. 5 shows the intermediate orbits in a four-impulse rendezvous problem, separated in the two distribution types mentioned. The variance of the Gaussian distribution $\sigma^{2}$ is a vector as $\sigma^{2}=\left[\sigma_{a}^{2} ; \sigma_{e}^{2} ; \sigma_{i}^{2} ; \sigma_{\Omega}^{2} ; \sigma_{\omega}^{2} ; \sigma_{\theta}^{2} ; \sigma_{t}^{2}\right]$, representing different variances for each type of variables in the optimization. Regarding the proposed approach, the input vector $x$ contains the orbital elements of the intermediate transfer trajectories along with the Lambert problem variables in each stage. Since the scale of variables in the input vector is different, the variances should be selected properly as constant values, or as the functions of some characteristics from initial orbit $p_{0}$ and final orbit $p_{f}$ in the space rendezvous 
mission.

\subsection{Automatic Parameter Tuning}

Since the objectives $J_{f}, J_{v}$ and $J_{t}$ have different types and scales, $\zeta$ and $\xi$ should be carefully tuned for each rendezvous problem. Also, the variance vector of variables $\sigma^{2}$ used in Eq. 21 needs to be tuned according to a feedback variable from the rendezvous problem. Rather than the seeding technique and the optimization algorithm, the parameters $\zeta$ and $\xi$ are also tuned as functions of the rendezvous problem itself. Obviously, one selection of these parameters for a specific rendezvous mission does not necessarily result in the optimal solution in another mission. The issue is that depending on the shape and orientation of the initial and final orbits or in general the amount of difference between the orbital elements $\left(p_{0}\right.$ and $\left.p_{f}\right)$, the sensitivity of these objectives varies. For instance, these parameters should be somehow auto-tuned to prevent the algorithm from sacrificing the impulse violation in favor of time. If this happens, the impulses achieved are not feasible, regardless of the total transfer time. Regarding the impulse violation coefficient $\zeta$, an arbitrary parameter $\Gamma$, representing the difference of initial and final orbits as $\Gamma\left(p_{0}, p_{f}\right)$ is defined by the following formula:

$$
\Gamma=\frac{\left|a_{0}-a_{f}\right|}{3 R e}+\frac{\left|e_{0}-e_{f}\right|}{0.5}+\frac{\left|i_{0}-i_{f}\right|}{\pi}+\frac{\left|\Omega_{0}-\Omega_{f}\right|}{\pi}+\frac{\left|\omega_{0}-\omega_{f}\right|}{\pi}
$$

where $R e$ is the Earth radius. This formula has five terms, corresponding to each orbital element, divided by some scaling factors. These scaling factors are selected in order to have the same impact on the overall value of $\Gamma$. The proof of this claim is illustrated in Fig. 6 and Fig. 7.

Fig. 6 and Fig. 7 show the cumulative mean value $\mu_{\Gamma}$ and the standard deviation $\sigma_{\Gamma}$ of the related term in $\Gamma$ for $n$ instances of different pairs of space orbits as the rendezvous missions. The plots show that the scaling factors are adjusted fairly for uniform changes of every $\Gamma$ terms regarding each orbital element as they converge almost to a same value. Besides the difference of orbital 


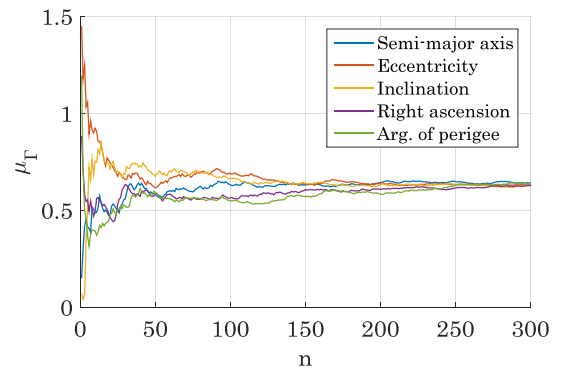

Figure 6: Cumulative mean value of $\Gamma$ terms.

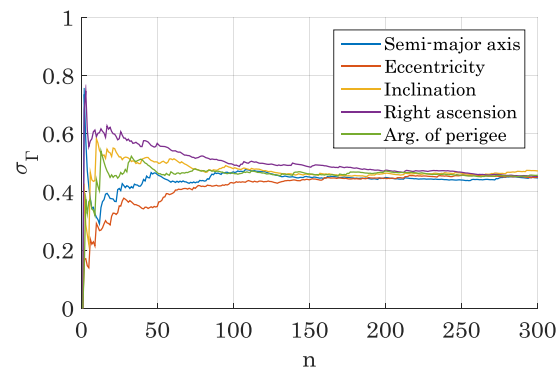

Figure 7: Cumulative standard deviation of $\Gamma$ terms.

elements, the minimum number of impulses required for the transfer according to the impulse limit is considered as a tuning feedback. This parameter, denoted by $\phi$, can be calculated after generating the feasible solution described in Section 3. Having the required impulses at orbit intersections $\varphi_{i, j}$, the total number of impulses is as:

$$
\phi=\sum_{i=1}^{N} \sum_{j=1}^{2} \varphi_{i, j}
$$

where $N$ is the total number of stages. The reason for considering this variable as feedback for tuning the algorithm parameter is that when the number of impulses increases, the likelihood of returning infeasible solutions by the algorithm will be higher. Utilizing this parameter, the variance vector of decision variables used in Eq. 21 is tuned as:

$$
\sigma^{2}=\left[\begin{array}{lllllll}
\sigma_{a}^{2} & \sigma_{e}^{2} & \sigma_{i}^{2} & \sigma_{\Omega}^{2} & \sigma_{\omega}^{2} & \sigma_{\theta}^{2} & \sigma_{t}^{2}
\end{array}\right]^{\prime}=\chi\left(1-e^{-\beta \phi}\right)
$$

where vector $\chi$ includes coefficients for each type of optimization variables. One selective value for $\chi$ is 0.1 for eccentricity, 180 for true anomalies, 5000 for time, and 100 for the rest of the optimization variables. Also, the value of 0.1 for $\beta$ adjusts the variances into a more comprehensive amount. Such a selection scales the variances to have the optimum distribution of the generated near optimal solutions. Also, regarding the obtained variables, the parameter $\zeta$ is defined as: 


$$
\zeta=\Gamma+\ln (\phi)
$$

This tuning method adjusts the impulse violation according to the complexity of the space mission and the number of stages which itself varies according to the given impulse limit. The coverage of this modeling is illustrated in Fig. 8.

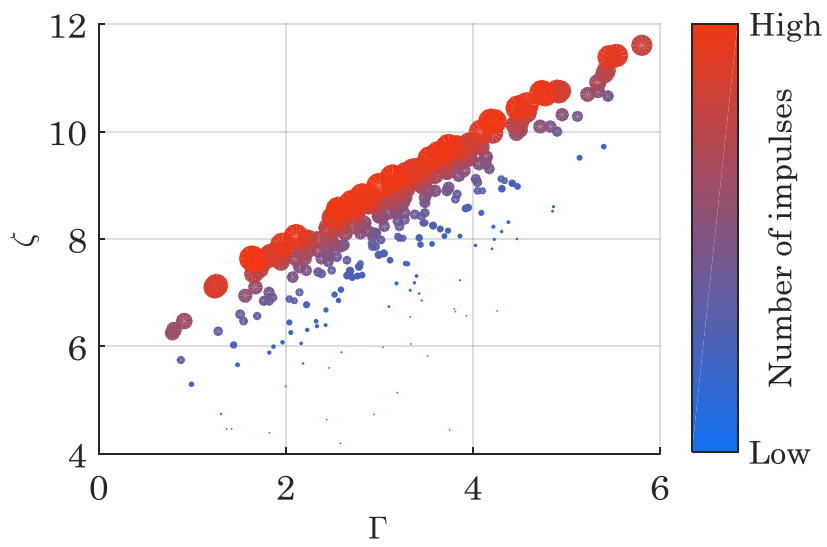

Figure 8: Impulse violation weighting coefficient coverage.

Fig. 8 shows the variation of $\zeta$ corresponding to the cost of impulse violation for the instances mentioned. A random impulse limit is considered for each space rendezvous mission, leading it to produce a different number of impulses for every long-range rendezvous. The points referring to high number of impulses are shown as big markers while smaller markers refer to the space missions with a low number of impulses. The proposed method makes the cost of impulse violation comparable to the fuel cost with respect to the complexity of the rendezvous mission and the impulse limit. Similarly, the weighting coefficient for the transfer time is defined as:

$$
\xi=\left(J_{t}^{*}\right)^{-1}
$$

where $J_{t}^{*}$ is the overall maneuver time of the multi-impulse transfer obtained within the analytical seeding phase of the algorithm. Since the algorithm at- 
tempts to find time-optimal solutions from the fuel-optimal region of the solution domain within the process of optimization, it is cruicial to have a dynamic coefficient for this cost with respect to the maximum transfer time between the initial individuals. Considering this value as the weighting coefficient for the time scale, the overall time will be scaled to one with respect to the initial seeds, making the value of $J_{t}$ comparable to the rest of the costs.

Once all of the parameters have been tuned, the proposed EA can be utilized to search for the best solution. The pseudo code for this strategy is presented in Algorithm 1.

As shown, the proposed strategy in dealing with the multi-objective space rendezvous ends up facing an optimization problem while having candidate solutions near the fuel-optimal region that satisfies the impulse limit. If the diversity of the populations is not satisfactory, such solutions are regenerated during the optimization process. Also, the parameters involved in the process are automatically tuned based on the orbital elements of the orbits and the required impulse limit.

\section{Numerical Simulations}

The proposed approach is investigated in several aspects in this section. First, a sample space rendezvous is solved considering two various impulse limits and the obtained orbital maneuvers are analyzed. The performance of the approach in finding the best minimum transfer time while having the near optimal fuel consumption without violating the impulse limit is studied when different impulse limits are considered for the same space rendezvous. Next, experiments are performed in which the approach is utilized in many different space rendezvous missions. Following the experiments, the performance of the proposed algorithm is compared with other standard EAs, indicating the superiority of the proposed algorithm due to the enhancements. 


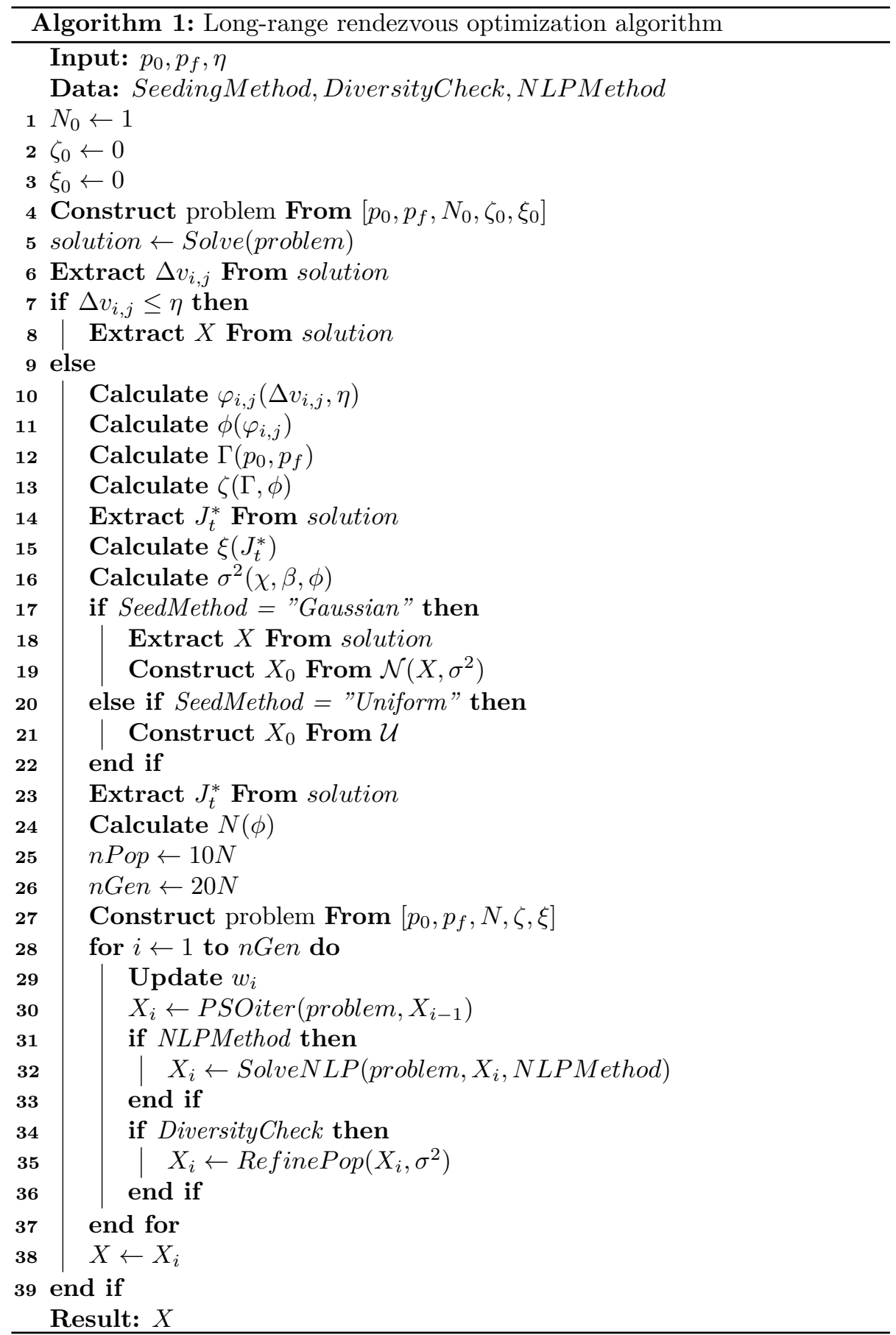




\subsection{Long-range Rendezvous}

A space rendezvous mission with two different impulse limits is evaluated. Consider an orbit to orbit rendezvous as in Table 1.

Table 1: Orbital elements of the orbit to orbit rendezvous

\begin{tabular}{lrr}
\hline Orbital elements & Initial & Final \\
\hline$a(\mathrm{~km})$ & 11300 & 32600 \\
$e$ & 0.2 & 0.5 \\
$i(\mathrm{deg})$ & 40 & 50 \\
$\Omega(\mathrm{deg})$ & 275 & 270 \\
$\omega(\mathrm{deg})$ & 280 & 265 \\
\hline
\end{tabular}

This space rendezvous is a non-coplanar transfer problem and two scenarios are considered with impulse limits of $\eta=200 \mathrm{~m} / \mathrm{s}$ and $\eta=50 \mathrm{~m} / \mathrm{s}$. As for the first step, disregarding the impulse limit and the transfer time, the best solution found for the optimal two-impulse transfer with minimum $\Delta v$ is the one that starts at $\vec{r}_{i}=[-3887 ;-7694.2 ;-3811.9] \mathrm{km}$ on the initial orbit and finishes at $\vec{r}_{f}=[4454.4 ; 25862 ; 5308.5] \mathrm{km}$ on the final orbit. This solution is obtained with the NLP method described in the previous section with few iterations. This transfer takes 24157 seconds with the total $\Delta v$ of $2.1353 \mathrm{~km} / \mathrm{s}$. Considering this solution as the fuel-optimal transfer, the analytical multi-impulse transfer is extracted and seeded to the developed hybrid self-adaptive algorithm. The algorithm parameters are tuned automatically based on the multi-impulse solution and the orbital parameters of the initial and final orbits as tabulated in Table 2 .

Table 2: Auto-tuned parameters of the self-adaptive algorithm

\begin{tabular}{lllllllllll}
\hline & $\phi$ & $\zeta$ & $\xi$ & $\sigma_{a}^{2}$ & $\sigma_{e}^{2}$ & $\sigma_{i}^{2}$ & $\sigma_{\Omega}^{2}$ & $\sigma_{\omega}^{2}$ & $\sigma_{\theta}^{2}$ & $\sigma_{t}^{2}$ \\
\hline$\eta=0.20$ & 12 & 4.36 & $2.40 \times 10^{-6}$ & 77.79 & 0.0419 & 3.88 & 1.94 & 5.82 & 125.79 & 3494 \\
$\eta=0.05$ & 44 & 5.66 & $7.27 \times 10^{-7}$ & 109.95 & 0.0592 & 5.48 & 2.74 & 8.23 & 177.79 & 4938 \\
\hline
\end{tabular}

The impulse discretization is performed at the intersections. Having the impulses at each intersection, the required number of divisions $(\varphi)$ are calculated using Eq. 7. It gives 6 divisions at each intersection for the first case and 23 and 21 divisions for the second case based on the desired impulse limit. Based 


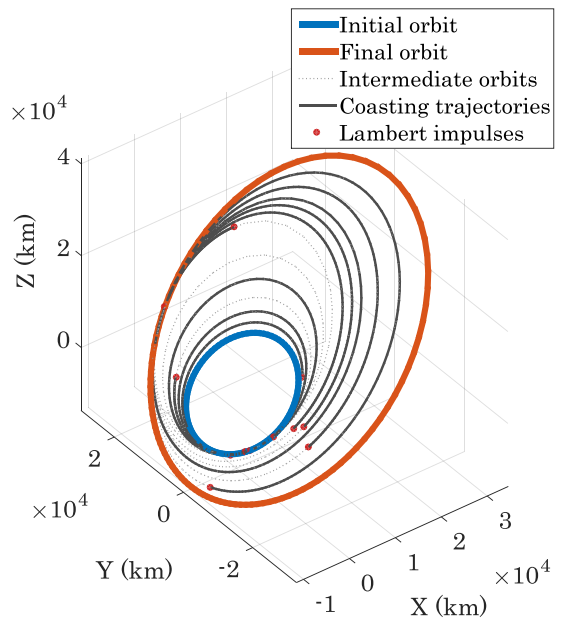

Figure 9: Optimized multi-impulse orbit rendezvous $(\eta=200 \mathrm{~m} / \mathrm{s})$.

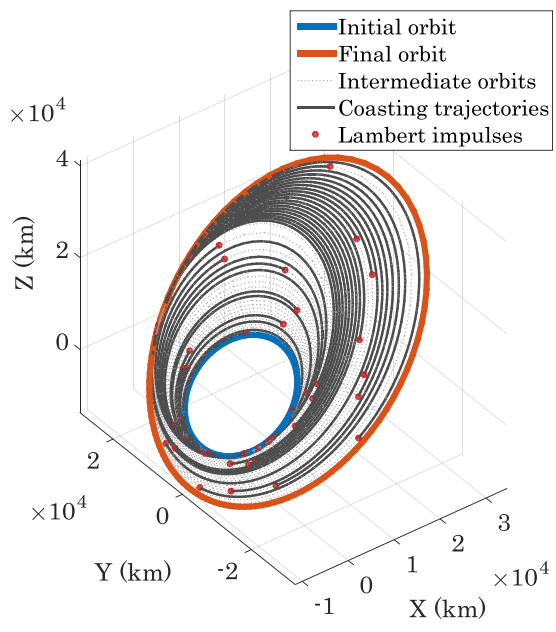

Figure 10: Optimized multi-impulse orbit rendezvous $(\eta=50 \mathrm{~m} / \mathrm{s})$.

on the obtained number of divisions in each intersections, the total number of impulses $\phi$ are achieved for each case using Eq. 23. The parameter $\Gamma$ representing the difference of orbits is 1.8799 for this transfer based on Eq. 22. Then, the scalarization coefficient $\zeta$ is obtained from Eq. 25 for both cases. Besides, the variances of optimization variables in $\sigma^{2}$ vector can be obtained from Eq. 24 Also, division of the impulses yields the multi-impulse transfers with the overall mission duration of 4.8214 days and 15.905 days for $\eta=200 \mathrm{~m} / \mathrm{s}$ and $\eta=50 \mathrm{~m} / \mathrm{s}$ respectively. By having these transfer times from the initial solution, the scalarization coefficient $\xi$ can be calculated from Eq. 26. Having all of the tuned parameters, the main problem can be tackled by the presented approach. The best time-optimal solutions are obtained with respect to the tuned parameters utilizing the developed self-adaptive algorithms. 3D visualization of the obtained transfers are depicted in Fig. 9 and Fig. 10.

In these maneuvers, the spacecraft travels between the stages which are optimized to have minimum overall transfer time, resulting in different Lambert problems in each revolution. The sequence of intermediate orbits along with the solution of the Lambert problem in each stage is optimized in order to minimize the overall transfer time. As shown in the figures, a lower impulse limit generally 
yields longer maneuver with more revolutions and impulses. The variations of impulses for both maneuvers are depicted in Fig. 11.

As shown, the impulse limits are satisfied for both maneuvers, leading to the conclusion that the weighting coefficients are almost properly tuned using the proposed method. Usually the untuned parameters lead the algorithm to sacrifice the impulse limit in the favor of transfer time which is not desired in the current concept. The optimized variables, such as solutions to the Lambert problems, can be plotted for either case. As previously mentioned, the problem input vector includes the true anomalies of the points where the spacecraft travels between two sequential stages. Their optimized values for two cases are illustrated in Fig. 12 and Fig. 13.

In these figures, the optimized anomalies are plotted during the overall orbital maneuver. Small points refer to the jumps at the beginning of the maneuver while bigger points are related to the impulses near the end of the mission. Comparing the location of the optimized true anomalies in the plots for two cases indicates that two potential regions can be identified as the near-optimal region for true anomalies between Lambert problems.

Regarding the obtained solution, the time histories of orbital elements can be simulated for the spacecraft as it travels from the initial orbit to the final orbit. The variation of orbital elements are shown in Fig. 14 to Fig. 17. Several

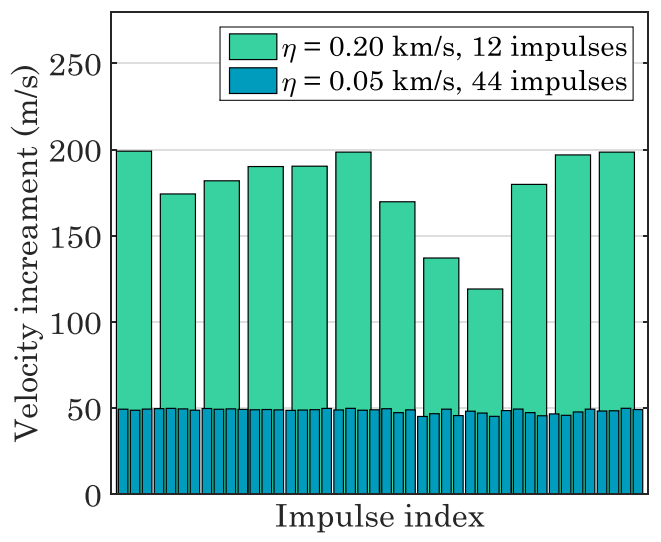

Figure 11: Sequence of impulses in multi-impulse long-range rendezvous. 


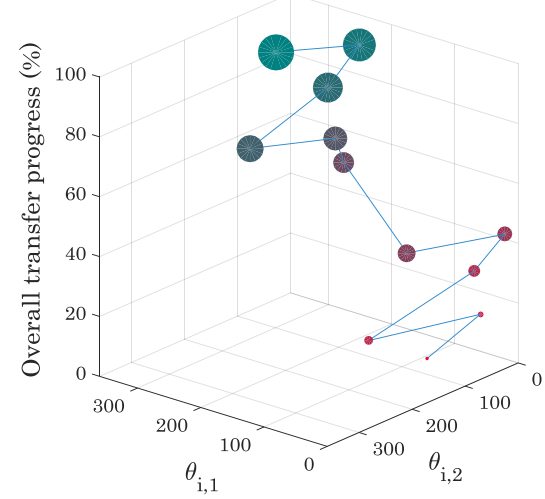

Figure 12: True anomalies of the Lambert problem $(\eta=200 \mathrm{~m} / \mathrm{s})$.

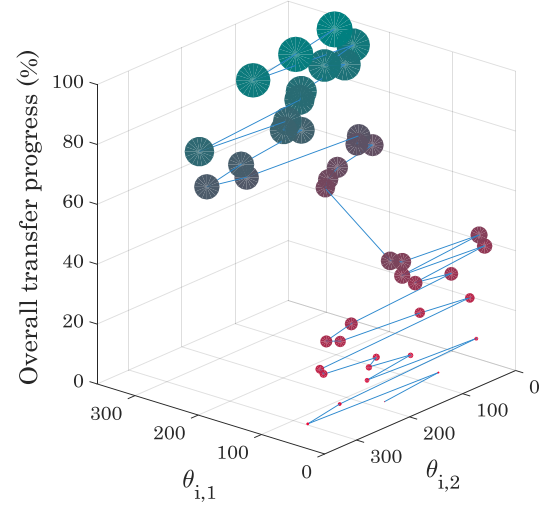

Figure 13: True anomalies of the Lambert problem $(\eta=50 \mathrm{~m} / \mathrm{s})$.

observations can be inferred from the time histories of orbital elements. The non-linear variation of the elements shows that considering the linear variation of elements is generally not an optimal choice, which is a confirmation for the practicality of the proposed method as it is actually a shape-based approach. Another observation is the time distance between two sequential impulses in the results. According to the variation of elements, this time distance increases as the spacecraft reaches the final orbit in both cases. This increment is in agreement with the variation of $a(t)$. Since the semi-major axis of the initial orbit is less than the final orbits, the time of one revolution will increase as the

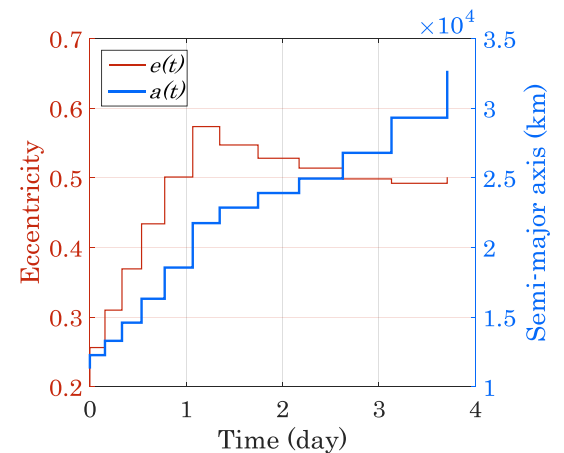

Figure 14: Time histories of semi-major axis and Eccentricity $(\eta=200 \mathrm{~m} / \mathrm{s})$.

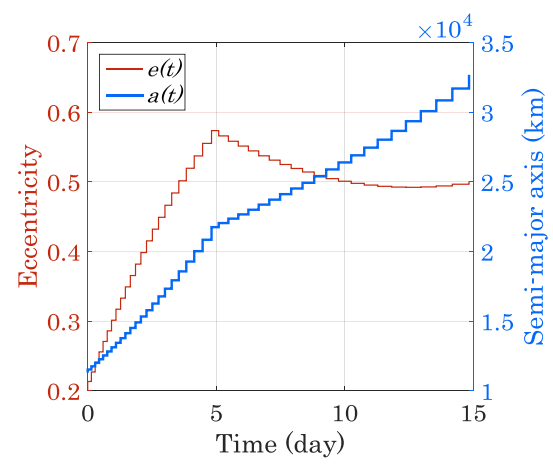

Figure 15: Time histories of semi-major axis and Eccentricity $(\eta=50 \mathrm{~m} / \mathrm{s})$. 




Figure 16: Time histories of inclination, Arg. of Perigee and RAAN $(\eta=200 \mathrm{~m} / \mathrm{s})$.

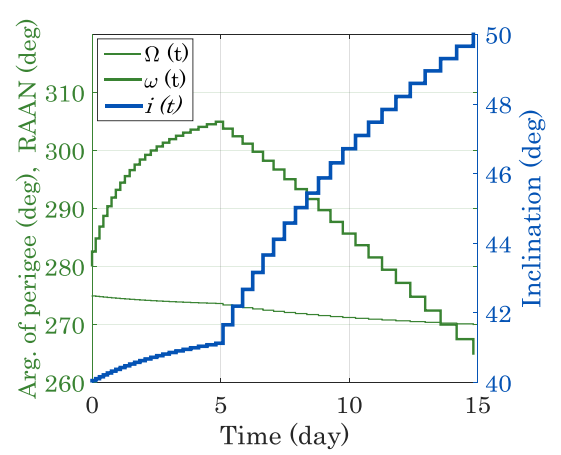

Figure 17: Time histories of inclination, Arg. of Perigee and RAAN $(\eta=50 m / s)$.

spacecraft travels between the intermediate orbit. This is due to the fact that the orbital period depends only on the semi-major axis in each stage.

The solutions can be also analyzed when various impulse limits are considered for a space rendezvous. As the impulse limit decreases, the tendency of the problem shifts to complicated solution domains, which are more difficult to optimize. Table 3 shows the characteristics of the previously defined space rendezvous problem considering various impulse limits.

In this Table, the entire process of tuning the parameters, generating nearoptimal feasible solutions, and solving the problem is performed for each impulse limit. First, the proposed simple feasible solution is obtained initially for each impulse limit, and the transfer times that were obtained before using the main algorithm are presented in the table. Then, the main algorithm is utilized to find the best solution based on the initial feasible solution. The obtained solutions are presented in the table to make a comparison regarding the difference between the transfer times. According to the results from the optimizations, the absolute improvement of the objective related to transfer time varies from case to case. Fig. 18 illustrates a representation of the best solution found so far regarding each impulse limit.

In this representation, the best 1000 solutions are saved during every optimization and are plotted altogether to give an insight to the near-optimal region. 
Table 3: Characteristics of space rendezvous problems.

\begin{tabular}{lllllllll}
\hline Impulse limit (m/s) & 300 & 250 & 200 & 150 & 100 & 75 & 50 & 25 \\
\hline Optimization variables & 59 & 75 & 91 & 115 & 179 & 235 & 347 & 691 \\
Initial transfer time (day) & 4.088 & 4.536 & 4.821 & 6.899 & 10.304 & 13.123 & 15.906 & 36.922 \\
Best transfer time (day) & 2.301 & 2.992 & 3.710 & 4.673 & 7.466 & 9.854 & 14.828 & 29.701 \\
\hline
\end{tabular}

For each unique impulse limit, the best solution is considered as the target and the relative distance of the rest of the solutions, denoted by $d_{i}$, is computed as:

$$
d_{i}=\frac{x_{i}-x^{*}}{\max _{i \in\{1, \ldots, n\}}\left[x_{i}-x^{*}\right]}
$$

where $n$ is the number of solutions, $i$ is the index of each solution, $x_{i}$ is the input vector of each solution, and $x^{*}$ is the best solution so far. Having, $d_{i}$ vector for each solution, the euclidean distance for that solution, denoted by $D_{i}$, can be computed as:

$$
D_{i}=\sqrt{\sum_{j=1}^{k} d_{i}(j)^{2}}
$$

where $k$ here is the size of the input vector which varies from case to case in the current analysis. Regarding the distances, the two local optima regions can be identified, which is very near the global best solution, leading to conclusion that the optimization process successfully identified the solution within the actual near-optimal region. As the impulse limit decreases, the absolute value of the
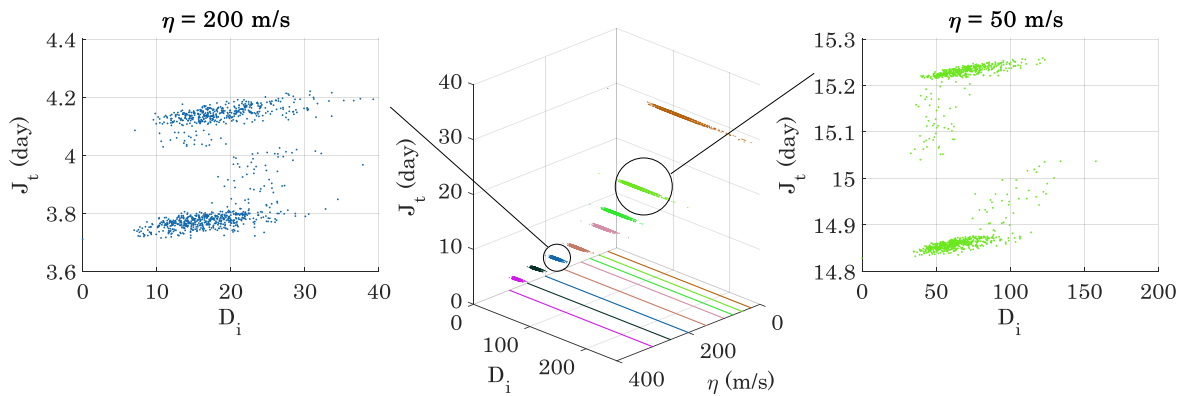

Figure 18: Euclidean distances of the best solutions in minimum-time minimum-fuel rendezvous. 
overall transfer time increases. However, it does not have a linear behavior with respect to the given limits. The distribution of points also indicates that the gradient of the solution domain within the near-optimal region is higher in the low impulse limit in comparison to the high impulse limit, confirming that the problem is more difficult to deal with in the first case. The absolute amount of solution improvement within the selected solutions is different regarding the results. In order to have an insight about the relative percentage of the improvement, the scaled values of the objectives convey more comprehensible data regarding the percentage of the improvement. One representation of the relative improvement of the selected solutions is shown in Fig. 19.

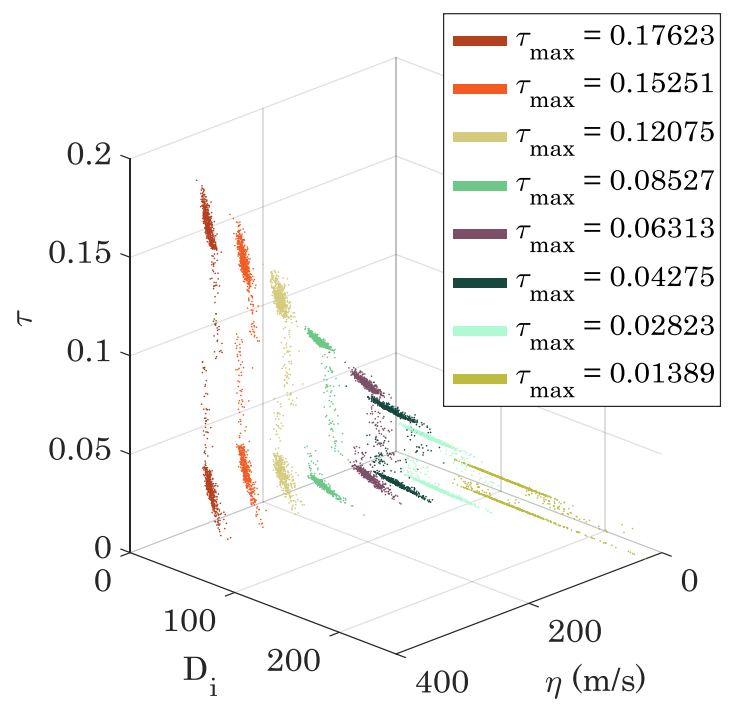

Figure 19: Relative objective improvement for various impulse limits.

Here, $\tau$ is the relative objective improvement calculated by:

$$
\tau=\frac{J^{*}-J}{J^{*}}
$$

where $J_{t}^{*}$ is the total transfer time obtained by the simple feasible solution. Comparing the $\tau$ values indicates that, although the absolute amount of the improved objective in impulsive rendezvous with very low impulse limit is high, 
the relative improvement is lower than those with higher impulse limits. This is the observation which confirms that the complexity of the optimization problem significantly increases and less improvement is gained by the algorithm as more impulses are taken into account for the space rendezvous.

As stated, the algorithm utilizes the analytical solution in two ways. First is the initial seeding in which the initial population is generated based on nearoptimal region according to the obtained feasible solution. The second one is a trigger during the optimization process which eliminates similar individuals and regenerates them based on a method the same as the initial seeding. The initial seeding of the algorithm and regeneration of individuals during the optimization process is handled by $\epsilon$ as described in this approach. This parameter specifies the balance between the Gaussian distribution and the uniform distribution when generating new individuals. The quality of the final solution achieved by the algorithm depends on the choice of $\epsilon$. Comparing the best solutions achieved with different values of $\epsilon$ considering various impulse limits is a matter of interest as it shows the rather optimal value of these parameters in different rendezvous problems. An illustration of such a comparison is shown in Fig. 20.

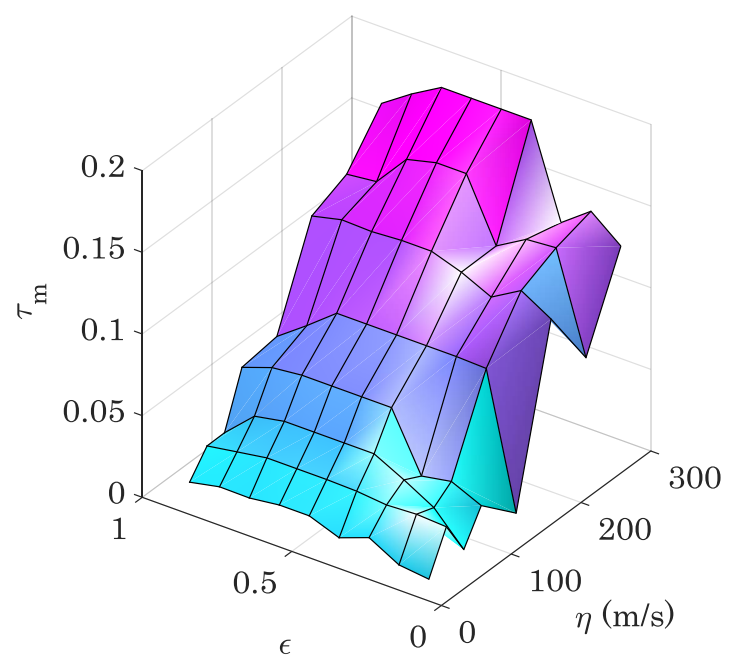

Figure 20: Average performance of the algorithm in 20 runs.

In this analysis, the problem is solved for each impulse limit considering 
different values for $\epsilon$. In each case, the optimization is carried out 10 times and all the objective improvements $\left(\tau_{\max }\right)$ are stored. Then, the average of improvement is calculated as:

$$
\tau_{m}=\frac{1}{n_{r}} \sum_{i=1}^{n_{r}} \tau_{\max }(i)
$$

where $n_{r}$ is the total number of optimization runs for each case, $\tau_{\max }(i)$ is the improvement in the $i$ th run. The surface illustrating the average performance of the algorithm shows that typically a value of 0.4 to 0.6 results in almost the maximum performance for all of the cases. It can also be concluded that in the rendezvous missions with higher impulse limits, the performance is affected more by the $\epsilon$ in comparison to missions with low impulse limits.

\subsection{Empirical Experiments}

When comparing one metaheuristic to another in a spacecraft trajectory optimization problem, it is crucial to perform benchmark tests using a suite of standard problems [10]. In order to make a practical comparison between algorithms, it is important to consider several factors. These factors include testing a large suite of instances of the rendezvous problems, using the same initial guess, and comparing them based on the same convergence criteria. To achieve this end, 100 instances of orbit to orbit rendezvous are tested by the proposed approach and some standard evolutionary algorithm. The orbital elements of the initial and final orbits are plotted in Fig. 21.

According to Fig. 21, the orbital elements are uniformly distributed as $6600 \mathrm{~km}<a_{i}, a_{f}<50000 \mathrm{~km}, 0<e_{i}, e_{f}<0.8,0^{\circ}<i_{i}, i_{f}<90^{\circ}, 0^{\circ}<\Omega_{i}, \Omega_{f}<$ $360^{\circ}$ and $0^{\circ}<\omega_{i}, \omega_{f}<360^{\circ}$. The elements are considered to satisfy the conditions as $-5000 \mathrm{~km}<\Delta a<5000 \mathrm{~km},-0.4<\Delta e<0.4,-30^{\circ}<\Delta i<30^{\circ}$, $-30^{\circ}<\Delta \Omega<30^{\circ},-30^{\circ}<\Delta \omega<30^{\circ}$. A random impulse limit is dedicated to each space mission within the range of $50 \mathrm{~m} / \mathrm{s}<\eta<500 \mathrm{~m} / \mathrm{s}$, resulting in problems with different number of variables and complexities. In Fig. 21, larger impulse limits are plotted with big markers while smaller ones represent space 

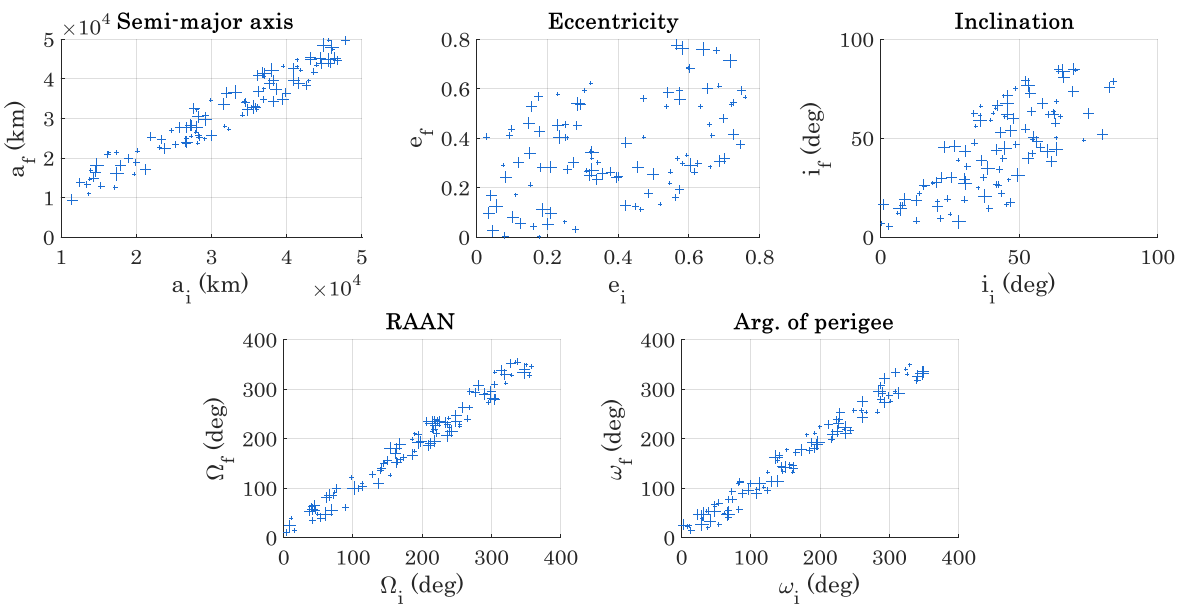

Figure 21: Distribution of orbital elements for empirical experiments.

rendezvous missions with lower impulse limits.

The algorithm presented has many features for robustness and self adaptiveness including hybridization with NLP, adaptive weighting coefficient, regeneration of individuals based on near fuel-optimal region, dynamic damping ratio, and analytical seeding based on discretization of orbit intersections. Therefore, numerous versions of this approach can be implemented by enabling or disabling any of these features. They will have a different effect on the obtained solution. However, due to brevity in this article, only three types of the approach are implemented and compared with other EAs. The first one is the Hybrid Self-Adaptive Evolutionary Algorithm (HSAEA), which is the full developed algorithm with all of the improvements. The second is the Self-Adaptive Evolutionary Algorithm (SAEA-I), which is the same as HSAEA but no hybridization with NLP is applied during the optimization process. The third one is another reduced version of the Self-Adaptive Evolutionary Algorithm (SAEA-II), which is the same as SAEA-I, but no regeneration of individuals for diversity correction is used during the optimization process. On the other hand, all these three versions benefit from the tuning of the parameters based on the characteristics of the rendezvous problem and dynamic damping ratio. Standard Particle 
Swarm Optimization (PSO) and Genetic Algorithm (GA) are also taken into account for comparison.

Since each space rendezvous mission with a distinct impulse limit generates a different optimization problem, the problems will have different complexities and a different number of optimization variables due to the various necessary numbers of impulses. Therefore, the common parameters of the algorithms have been chosen in such a way that a fair comparison between the performance of each algorithm is achieved. For each problem, the number of populations and the number of generations for all algorithms are set as $10 n$ and $20 n$ respectively, where $n$ is the number of optimization variables for that space rendezvous mission. Regarding the three versions of the proposed approach, HSAEA, SAEA-I and SAEA-II, all algorithm parameters are tuned automatically for each problem according to the presented process in Section 4. For PSO, the values of 1.8 and 2.0 are chosen for personal and global learning coefficients respectively. Also, the inertial weight is set to 1.0 with the damping ratio of 0.95 per generation. For GA, crossover percentage and crossover range factor are chosen as 0.6 and 0.3 respectively. Also, the mutation percentage and the mutation range are selected as 0.4 and 0.2 respectively. The reason for choosing these parameters for PSO and GA is that they are statistically shown to have the best performance for these algorithms in most of the space rendezvous missions with the current setup. Since the proposed algorithms benefits from the automatic tuning of parameters, the best settings of the two selected EAs are chosen for performance comparison. Although tuning the algorithm parameters is another optimization problem itself, the effort in this research is to use the best performance of PSO and GA for comparison as these values outperform other combinations in most instances of space rendezvous missions.

For this analysis, HIPATIA cluster setup of BCAM is used with 18 nodes including 624 cores (Processor Intel(R) Xeon(R) Gold $6140 \mathrm{CPU} @ 2.30 \mathrm{GHz}$ ) and 4352 GB RAM. Having 100 space rendezvous problems, each problem is solved with the 5 aforementioned algorithms (HSAEA, SAEA-I, SAEA-II, PSO, GA) and each algorithm is run 10 times. Therefore, a total number of 5000 jobs 
are submitted to the cluster to run the optimizations in parallel. The solutions obtained are saved for each algorithm and the best solution between all of the runs is assumed to be the global best solution for each rendezvous mission. Then, for each problem, the relative score of the all solutions obtained is calculated as:

$$
\gamma_{i, j}=\frac{J_{i, j}-J_{b e s t}}{J^{*}-J_{b e s t}}
$$

where $J_{i, j}$ is the objective function obtained for the $i$ th run of the $j$ th algorithm $\left(0<i \leq N_{r}, 0<j \leq N_{a}\right)$, considering 10 runs $\left(N_{r}=10\right)$ for the 5 aforementioned algorithms $\left(N_{a}=5\right) . J^{*}$ is the objective function obtained by the simple feasible solution, and $J_{b e s t}$ is the best obtained solution between all of the runs, which is assumed to be the global best for the rendezvous mission as:

$$
J_{\text {best }}=\arg \min \left[J_{i, j}\right] \quad\left(i=1, \ldots, N_{r}, j=1, \ldots, N_{a}\right)
$$

The reason of considering such a type of score is that different rendezvous missions with various impulse limits will have different values of objective functions, and therefore it is difficult to make a comparison between different instances. This definition scales the performance of algorithms in each run into a dimensionless score between 0 and 1 , where 0 indicates that the algorithm successfully reached the global solution, while 1 shows that the algorithm did not make any improvement over the simple feasible solution and could not decrease the objective function within the optimization process. Any score value in this range shows how much improvement the algorithm obtained in reaching the global best solution. Having all of the scores for the algorithms, an insight to the overall performances can be achieved. Fig. 22 shows the performance comparison between the algorithms.

Fig. 22 indicates the absolute score of the algorithms applied to different space rendezvous problems. This figure shows the results for 5000 obtained scores (10 runs for each of the 5 algorithms in 100 instances). This score gives 


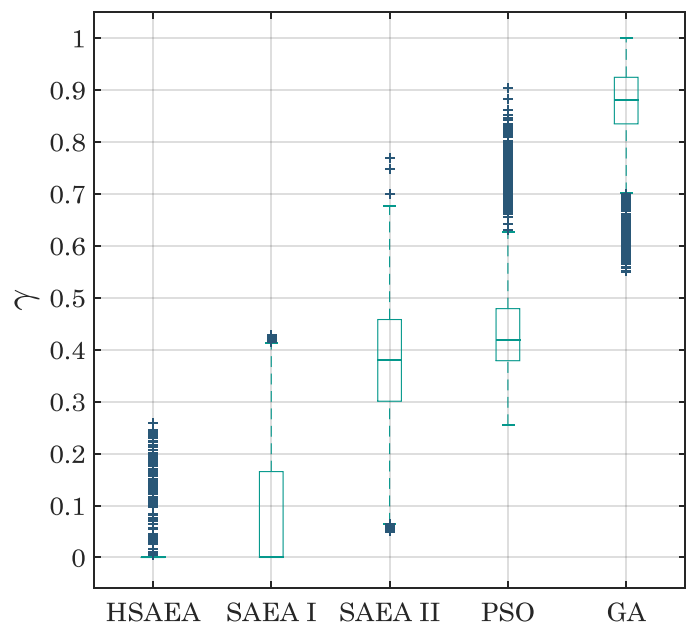

Figure 22: Absolute scores of the algorithms for multi-impulse space rendezvous.

an insight into the performance of the algorithms. However, it does not convey the average performance of the algorithms for each instance. The average performance of an algorithm is the mean value of all obtained solutions out of the optimization runs for that algorithm regarding a specific problem. It can be simply calculated as:

$$
\gamma_{j}^{\prime}=\frac{1}{N_{r}} \sum_{i=1}^{N_{r}} \gamma_{i, j}
$$

where $\gamma_{j}^{\prime}$ is the average performance of the $j$ th algorithm considering all optimization runs. The relative performances of the algorithms based on this score are illustrated in Fig. 23.

Regarding the results, the HSAEA has superior advantage over the rest of the cases, showing the effectiveness of the proposed algorithm when comparing to the standard EAs. Noticing the best scores for each algorithm indicates that only HSAEA and SAEA-I reached the scores of 0 (global best) while SAEA-II, PSO and GA failed to achieve the global best in any of the instances, leading to the conclusion that hybridization of the algorithm with the proposed NLP and regeneration of populations near the fuel-optimal region is necessary to achieve 


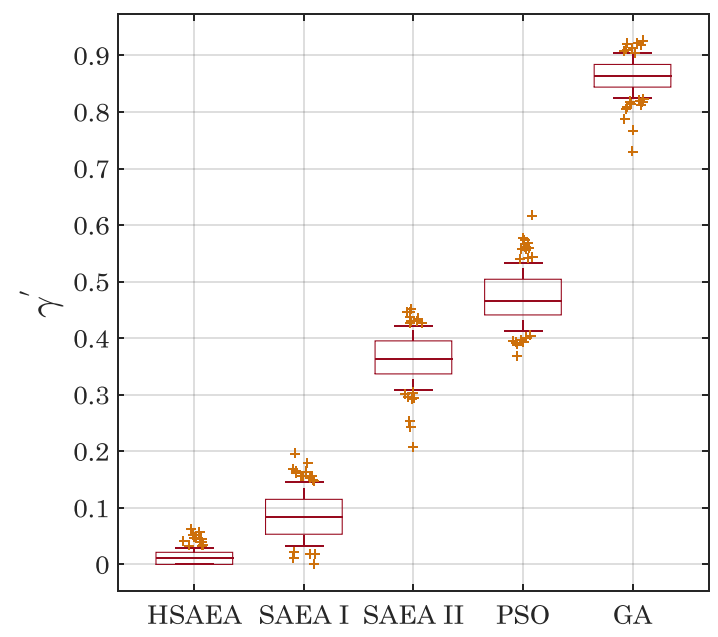

Figure 23: Relative scores of the algorithms for multi-impulse space rendezvous.

the global best. The comparison between HSAEA and SAEA-I also shows that both algorithms most likely have reached the global optimal solution in the majority of space rendezvous missions and the difference is the probability of reaching the optimal solution. Therefore, it can be implicitly concluded that hybridization with NLP increases the rate of reaching the global optimal solution in different optimization runs, while regeneration of populations near the fueloptimal region within the optimization process guarantees the convergence to the global optimal point. Comparing the results from SAEA-II and PSO shows that, although the general performance of two algorithms is almost the same, the SAEA-II has a slight advantage in some of the cases over PSO due to dynamic damping ratio. Also, the standard PSO itself outperforms the standard GA in all of the cases, leading to the conclusion that hybridization of GA with the proposed NLP probably does not outperform the presented HSAEA since the proposed HSAEA is generally based on an improved PSO.

While the effectiveness and reliability of the algorithms are compared by showing how close the algorithms get to the best solution, their efficiency can be evaluated by comparing their running time. For the sake of completeness in this research, the speed of the algorithms is measured and compared in ten 
selective instances of space rendezvous missions. Results are tabulated in Table 4 .

Table 4: Comparison of dimensionless running time (running time relative to the one associate with GA $\left.\left[s / s_{(G A)}\right]\right)$ of the algorithms, omitting the time of cost function evaluations.

\begin{tabular}{lllll}
\hline Instance & HSAEA & SAEA-I & SAEA-II & PSO \\
\hline 1 & 7.3198 & 2.0986 & 1.1328 & 1.0898 \\
2 & 6.2147 & 2.2233 & 1.1143 & 1.0165 \\
3 & 4.2777 & 2.0138 & 1.0457 & 1.0348 \\
4 & 5.2043 & 1.2076 & 1.0404 & 1.0274 \\
5 & 5.3826 & 1.3224 & 1.2031 & 1.0966 \\
6 & 4.3526 & 3.1734 & 1.1279 & 1.0967 \\
7 & 6.4117 & 2.4191 & 1.0880 & 1.0501 \\
8 & 7.1940 & 2.3722 & 1.1475 & 1.0825 \\
9 & 3.3919 & 2.5298 & 1.1231 & 1.0833 \\
10 & 4.0869 & 1.7127 & 1.1404 & 1.0682 \\
\hline Average & $\mathbf{5 . 3 8 3 6}$ & $\mathbf{2 . 1 0 7 3}$ & $\mathbf{1 . 1 1 6 3}$ & $\mathbf{1 . 0 6 4 6}$ \\
\hline
\end{tabular}

In this comparison, the running time of GA is considered as the base score for the speed of other algorithms, since GA has the minimum processing time in this set of algorithms. The time of objective function evaluations are neglected and the calculated running time for each algorithm is divided by the base time of GA for each instance. It has been shown that typical PSO has the least running time as it does not involve any modifications described in this research. SAEA-II, which benefits from automatic parameter tuning and dynamic damping ratio during optimization, requires slightly more time compared to PSO. The comparison of speed between SAEA-I and SAEA-II shows the effect of diversity correction within the optimization. Since regeneration of new individuals is triggered when the diversity of the population is not satisfactory, its occurrence is largely due to the randomness of movement of individuals. Finally, the HSAEA has the slowest process as the NLP employment for improving the quality of the individuals requires more iterations and calculations. It can be observed that the running time is significantly high for HSAEA in comparison to the other algorithms. However, it has the highest possibility to end up finding the best possible solution. 


\section{Conclusions}

The long-range space rendezvous problem was addressed using the proposed evolutionary discretized Lambert approach in this paper. In this research, all objectives, including fuel, time and impulse limit were considered. The proposed approach attempts to find the global optimal solutions which satisfy the impulse limit while minimizing the fuel and time simultaneously. The fueloptimal region is identified by means of a discretized approach in which the minimum necessary number of impulses is achieved by dividing the impulses within the intersection of trajectories between two sequential Lambert problems. The obtained solution is utilized to generate near-optimal solutions for seeding the optimization algorithm in order to find the optimal sequence of impulses which results in minimum-time minimum-fuel orbit transfer. The developed self-adaptive algorithm is a hybrid method, combined with auto-tuning techniques and an intelligent individual refinement procedure. The approach is used to solve some multi-impulse rendezvous problems. Results confirm the feasibility of the approach and show that it successfully improves the the optimality of the solution in terms of fuel and time without violating the impulse limit. The percentage of optimality is significant when the approach is used with the developed self-adaptive algorithm. Also, the robustness of the approach is tested by applying the proposed method in different types of rendezvous problems. Comparing the obtained results with the output of standard non-adaptive algorithms indicates the superiority of the developed algorithm. It has been shown that the probability of reaching optimal solutions is significantly higher with the current approach in comparison to other algorithms, since the approach benefits from self-adaptive tuning and hybridization. However, the running time burden is relatively high when the NLP is involved with the optimization process. Increasing the optimality of the solution and the robustness of the technique by means of considering more tuning techniques will be the next step in future research. 


\section{Acknowledgments}

This research is supported by La Caixa Fellowship, the Basque Government through the BERC 2018-2021, Elkartek programs, and by Spanish Ministry

of Economy and Competitiveness MINECO: BCAM Severo Ochoa excellence accreditation SEV-2017-0718, TIN2016-78365R and TIN2017-82626R projects.

\section{References}

[1] H. Lei, B. Xu, L. Zhang, Trajectory design for a rendezvous mission to earth's trojan asteroid 2010tk7, Advances in Space Research 60 (11) (2017) 2505-2517. doi:10.1016/j.asr.2017.09.020.

URL https://doi.org/10.1016/j.asr.2017.09.020

[2] M. Pontani, Optimal low-thrust hyperbolic rendezvous for earth-mars missions, Acta Astronauticadoi:10.1016/j.actaastro.2019.02.020.

URL https://doi.org/10.1016/j.actaastro.2019.02.020

[3] J. Li, Revisiting the fuel-optimal four-impulse rendezvous problem near circular orbits, Advances in Space Research 60 (10) (2017) 2181-2194. doi: $10.1016 / j$. asr. 2017.08 .035 .

URL https://doi.org/10.1016/j.asr.2017.08.035

[4] K. Kitamura, K. Yamada, T. Shima, Minimum energy coplanar orbit transfer of geostationary spacecraft using time-averaged hamiltonian, Acta Astronautica 160 (2019) 270-279. doi:10.1016/j.actaastro.2019.04.033. URL https://doi.org/10.1016/j.actaastro.2019.04.033

[5] H. Chen, C. Han, Y. Rao, J. Yin, X. Sun, Algorithm of relative lambert transfer based on relative orbital elements, Journal of Guidance, Control, and Dynamics (2019) 1-10doi:10.2514/1.g003348.

URL https://doi.org/10.2514/1.g003348

[6] Y. Luo, J. Zhang, G. Tang, Survey of orbital dynamics and control of space rendezvous, Chinese Journal of Aeronautics 27 (1) (2014) 1-11. doi: 
$10.1016 / j \cdot c j a .2013 .07 .042$.

URL https://doi.org/10.1016/j.cja.2013.07.042

[7] H. D. Curtis, Orbital mechanics for engineering students, ButterworthHeinemann, 2013.

[8] Z. Zheng, J. Guo, E. Gill, Distributed onboard mission planning for multisatellite systems, Aerospace Science and Technology 89 (2019) 111-122. doi:10.1016/j.ast.2019.03.054.

URL https://doi.org/10.1016/j.ast.2019.03.054

[9] M. Pontani, B. A. Conway, Particle swarm optimization applied to space trajectories, Journal of Guidance, Control, and Dynamics 33 (5) (2010) 1429-1441. doi:10.2514/1.48475.

URL https://doi.org/10.2514/1.48475

[10] A. Shirazi, J. Ceberio, J. A. Lozano, Spacecraft trajectory optimization: A review of models, objectives, approaches and solutions, Progress in Aerospace Sciences 102 (2018) 76-98. doi:10.1016/j.paerosci.2018. 07.007.

URL https://doi.org/10.1016/j.paerosci.2018.07.007

[11] A. A. Quarta, G. Mengali, Simple solution to optimal cotangential transfer between coplanar elliptic orbits, Acta Astronautica 155 (2019) 247-254. doi: $10.1016 / j$. actaastro.2018.12.007.

URL https://doi.org/10.1016/j.actaastro.2018.12.007

[12] J. Zhang, G. jin Tang, Y. zhong Luo, Optimization of an orbital longduration rendezvous mission, Aerospace Science and Technology 58 (2016) 482-489. doi:10.1016/j.ast.2016.09.011.

URL https://doi.org/10.1016/j.ast.2016.09.011

[13] M. S. Mohammadi, A. Naghash, Robust optimization of impulsive orbit transfers under actuation uncertainties, Aerospace Science and Technology 
85 (2019) 246-258. doi:10.1016/j.ast.2018.11.026.

URL https://doi.org/10.1016/j.ast.2018.11.026

[14] H. Ma, S. Xu, Optimization of bounded low-thrust rendezvous with terminal constraints by interval analysis, Aerospace Science and Technology 79 (2018) 58-69. doi:10.1016/j.ast.2018.05.031.

URL https://doi.org/10.1016/j.ast.2018.05.031

[15] K. Lee, C. Park, S.-Y. Park, Near-optimal continuous control for spacecraft collision avoidance maneuvers via generating functions, Aerospace Science and Technology 62 (2017) 65-74. doi:10.1016/j.ast.2016.11.026.

URL https://doi.org/10.1016/j.ast.2016.11.026

[16] G. Zhang, D. Zhou, D. Mortari, T. A. Henderson, Analytical study of tangent orbit and conditions for its solution existence, Journal of Guidance, Control, and Dynamics 35 (1) (2012) 186-194.

[17] G. Zhang, G. Ma, D. Li, Two-impulse transfer between coplanar elliptic orbits using along-track thrust, Celestial Mechanics and Dynamical Astronomy 121 (3) (2015) 261-274.

[18] S. Mitani, H. Yamakawa, Continuous-thrust transfer with control magnitude and direction constraints using smoothing techniques, Journal of Guidance, Control, and Dynamics 36 (1) (2012) 163-174.

[19] M. Avendaño, V. Martín-Molina, J. Martín-Morales, J. Ortigas-Galindo, Algebraic approach to the minimum-cost multi-impulse orbit-transfer problem, Journal of Guidance, Control, and Dynamics 39 (8) (2016) 1734-1743. doi:10.2514/1.g001598.

URL https://doi.org/10.2514/1.g001598

[20] G. Zhang, X. Cao, D. Zhou, Two-impulse cotangent rendezvous between coplanar elliptic and hyperbolic orbits, Journal of Guidance, Control, and Dynamics 37 (3) (2014) 964-970. doi:10.2514/1.62477.

URL https://doi.org/10.2514/1.62477 
[21] P. V. Arlulkar, S. D. Naik, Dynamical approach for optimal two-impulse rendezvous between elliptic orbits, Journal of Guidance, Control, and Dynamics 37 (3) (2014) 1008-1015. doi:10.2514/1.61823.

URL https://doi.org/10.2514/1.61823

[22] Z. Yang, Y.-Z. Luo, J. Zhang, G.-J. Tang, Homotopic perturbed lambert algorithm for long-duration rendezvous optimization, Journal of Guidance, Control, and Dynamics 38 (11) (2015) 2215-2223. doi:10.2514/1. g001198.

URL https://doi.org/10.2514/1.g001198

[23] E. Taheri, J. L. Junkins, Generic smoothing for optimal bang-off-bang spacecraft maneuvers, Journal of Guidance, Control, and Dynamics 41 (11) (2018) 2470-2475.

[24] J. Shimoun, E. Taheri, I. Kolmanovsky, A. Girard, A study on GPUenabled lambert's problem solution for space targeting missions, in: 2018 Annual American Control Conference (ACC), IEEE, 2018, pp. 664-669. doi:10.23919/acc.2018.8431478.

URL https://doi.org/10.23919/acc.2018.8431478

[25] C. F. Gauss, Theory of the Motion of the Heavenly Bodies Moving about the Sun in Conic Sections: A Translation of Gauss's" Theoria Motus." With an Appendix, Little, Brown and Company, 1857.

[26] G. Avanzini, A simple lambert algorithm, Journal of Guidance, Control, and Dynamics 31 (6) (2008) 1587-1594. doi:10.2514/1.36426.

URL https://doi.org/10.2514/1.36426

[27] D. Izzo, Revisiting lambert's problem, Celestial Mechanics and Dynamical Astronomy 121 (1) (2014) 1-15. doi:10.1007/s10569-014-9587-y.

URL https://doi.org/10.1007/s10569-014-9587-y

[28] S. L. Nelson, P. Zarchan, Alternative approach to the solution of lambert's 
problem, Journal of Guidance, Control, and Dynamics 15 (4) (1992) 10031009.

[29] O. Abdelkhalik, D. Mortari, N-impulse orbit transfer using genetic algorithms, Journal of Spacecraft and Rockets 44 (2) (2007) 456-460. doi: $10.2514 / 1.24701$.

URL https://doi.org/10.2514/1.24701

[30] Y.-Z. Luo, G.-J. Tang, Z.-G. Wang, H.-Y. Li, Optimization of perturbed and constrained fuel-optimal impulsive rendezvous using a hybrid approach, Engineering Optimization 38 (8) (2006) 959-973.

[31] S. A. Darani, O. Abdelkhalik, Convergence analysis of hidden genes genetic algorithms in space trajectory optimization, Journal of Aerospace Information Systems 15 (4) (2018) 228-238. doi:10.2514/1.i010564.

URL https://doi.org/10.2514/1.i010564

[32] M. Taherkhani, R. Safabakhsh, A novel stability-based adaptive inertia weight for particle swarm optimization, Applied Soft Computing 38 (2016) 281-295. doi:10.1016/j.asoc.2015.10.004.

URL https://doi.org/10.1016/j.asoc.2015.10.004

[33] J. Nocedal, S. Wright, Numerical optimization, Springer Science \& Business Media, 2006.

[34] M. R. Bonyadi, Z. Michalewicz, X. Li, An analysis of the velocity updating rule of the particle swarm optimization algorithm, Journal of Heuristics 20 (4) (2014) 417-452.

[35] J. Barrera, O. Álvarez-Bajo, J. J. Flores, C. A. Coello Coello, Limiting the velocity in the particle swarm optimization algorithm, Computación y Sistemas 20 (4) (2016) 635-645.

[36] M. Alhussein, S. I. Haider, Improved particle swarm optimization based on velocity clamping and particle penalization, in: 2015 3rd International 
Conference on Artificial Intelligence, Modelling and Simulation (AIMS),

IEEE, 2015, pp. 61-64. 